This item was submitted to Loughborough's Research Repository by the author.

Items in Figshare are protected by copyright, with all rights reserved, unless otherwise indicated.

\title{
Disturbance observer-based autonomous landing control of unmanned helicopters on moving shipboard
}

PLEASE CITE THE PUBLISHED VERSION

https://doi.org/10.1007/s11071-020-05915-w

PUBLISHER

Springer Science and Business Media LLC

VERSION

AM (Accepted Manuscript)

\section{PUBLISHER STATEMENT}

This paper was accepted for publication in the journal Nonlinear Dynamics and the definitive published version is available at https://doi.org/10.1007/s11071-020-05915-w.

\section{LICENCE}

All Rights Reserved

\section{REPOSITORY RECORD}

Yu, Xin, Jun Yang, and Shihua Li. 2020. "Disturbance Observer-based Autonomous Landing Control of Unmanned Helicopters on Moving Shipboard". Loughborough University.

https://hdl.handle.net/2134/16817869.v1. 


\title{
Disturbance observer-based autonomous landing control of unmanned helicopters on moving shipboard
}

\author{
Xin Yu · Jun Yang · Shihua Li
}

Received: date / Accepted: date

\begin{abstract}
In this paper, the autonomous landing control issue on moving shipboard is investigated for unmanned helicopters subject to disturbances. The issue is studied by stabilizing the error system of the helicopter and the shipboard. The landing process is divided into two phases, i.e., homing phase, where a hierarchical double-loop control scheme is developed such that the helicopter is forced to hover synchronously at a certain altitude over the shipboard, and landing phase, where a composite landing control scheme is proposed such that the helicopter lands vertically on the shipboard in synchronization with its attitudes. The velocity and acceleration information of the shipboard as well as lump disturbances are estimated through joint state and disturbance observers. The estimates are then incorporated into the baseline feedback controller, formulating composite active anti-disturbance landing control schemes. A continuous terminal sliding mode control method is proposed for the feedback controller design, which not only effectively mitigates the chattering of the control action, but also simplifies the design process of the controller. Numerical simulations demonstrate the effectiveness and superiorities of the proposed control schemes.
\end{abstract}

Keywords unmanned helicopters · landing control $\cdot$ moving shipboard · joint state and disturbance observer $\cdot$ continuous terminal sliding mode control

\section{Introduction}

Unmanned aerial vehicles (UAVs) have received a great deal of research, development and application for several decades [1]. Owing to the unique and significant abilities of vertical takeoff and landing, sideslip, backward flight and hovering, unmanned helicopters have been widely utilized to perform a variety of missions in various environments. On one hand, in all flight missions, autonomous landing is an essential stage and is prone to accidents, especially landing on moving targets. On the other hand, in all operating environments, maritime operating is very precarious and challenging due to the rough sea conditions

Xin Yu $\cdot$ Jun Yang $\cdot$ Shihua Li

School of Automation, Southeast University, Nanjing 210096, PR China

Key Laboratory of Measurement and Control of Complex Systems of Engineering, Ministry of Education, PR China

Xin Yu · Jun Yang

Shenzhen Research Institute, Southeast University, Shenzhen 518063, PR China

Jun Yang (corresponding author)

E-mail: j.yang84@seu.edu.cn 
such as low visibility, strong wind and turbulence, unpredictable motions of the shipboard, and narrow deck space [1]. For these two reasons, it is of great academic significance and research value to exploit autonomous landing schemes for helicopters on moving shipboard.

In recent decades, many researches have been carried out on autonomous landing of UAVs on moving targets. The focus of these researches is different, ranging from improving the algorithm of obtaining system information [2,3], optimizing control parameters [4,5], improving the accuracy of sensors [6], to improving control performances [8-16]. In terms of improving control performances, some linear control methods, such as proportional-integral-derivative control $[8,9]$, are proposed to achieve autonomous landing of UAVs in linear flight regimes. Since only specific flight regimes are considered and the complex nonlinear dynamics of UAVs are ignored, the landing performance under complex and changeable operating conditions is hardly to be satisfied. For better landing performance, many nonlinear control methods have been proposed, such as robust $H_{\infty}$ control [10,11], backstepping control [12,13], and sliding mode control (SMC) [14-16].

Among these nonlinear control methods, SMC method is an effective method due to its remarkable advantages in improving anti-disturbance performance and robustness of the system [18, 19]. As an important branch of SMC method, the terminal sliding mode control (TSMC) method has more advantages because it guarantees the finite-time convergence of the closed-loop system, which is vital for the shipboard landing helicopter system since a fast landing process reduces the exposure of the helicopter to environment disturbances and thus reduces their effects to a certain extent. However, due to the existence of the discontinuous sign function, the control action of traditional SMC/TSMC is discontinuous [14], which will lead to severe control chattering and large control amplitude. Generally speaking, there are two categories of feasible strategies to mitigate the chattering of the control action. The first category is to replace the sign function in the controller with continuous function [15]. For example, in [15], by replacing the sign function with fractional-power function, a continuous control is employed for the shipboard landing control such that the helicopter lands on the moving shipboard in fixed settling time. The second category is to make the sign function appear only in the derivative of the controller [18] such that the control action is continuous and the chattering is mitigated.

In the existing literature, many works achieve autonomous landing by stabilizing the relative motion between the UAV and the moving target [14,15,21-23]. To this end, the dynamics and full state information of the shipboard should be utilized, which generally are not available in reality due to the following technical constrains. Firstly, the motion of the moving shipboard is generally a six-degree-of-freedom (6-DOF) complex model, which is difficult to establish comprehensively. Secondly, even if most of the shipboard dynamics can be established through some methods, the process would be complex and expensive. In addition, it will cause additional errors due to the unmodeled shipboard dynamics. Thirdly, the measurement of shipboard's velocity and angular velocity increases the use of sensor modules, and the measured data may be not adequately accurate because of measurement noises. Based on the above analyses, this paper adopts the method of stabilizing the error system between the helicopter and shipboard to achieve autonomous landing, but does not need to establish the shipboard dynamics. What's more, the velocity and angular velocity of the shipboard are estimated based on known signals, which not only saves sensors but also improves the accuracy of the state information.

In addition, the sea condition is rough with intricate disturbances in shipboard landing process, which brings about adverse influences to the stability and landing performance of the helicopter system [1]. Although the disturbance can be suppressed through SMC method, this is a robust approach. Specifically, the gains of the controller are usually chosen to be larger than the upper bound of the disturbance, which is quite conservative and will lead to large control chattering. To this end, an effective way is to estimate the disturbance from measurable variables and to compensate the influences of them via a feedforward manner [24-26]. This is suitable for the controller design in this paper because the velocity and angle velocity of the shipboard as well as the lumped disturbances can be estimated together. In recent decades, some observers for estimating various kinds of disturbances have been proposed [27-31]. For example, 
in [27], a nonlinear disturbance observer is developed for disturbances generated by an exogenous system such that the estimation error system is globally exponentially stable. [28] proposes a high-order sliding mode observer (HOSMO) for a general class of disturbances, which provides finite-time convergence of the disturbance estimation error system. An innovative resonant extended state observer dedicated to harmonic disturbances is designed for 3-DOF torsional system in [30] and is extended for harmonic disturbances with unknown frequency in [31].

In this paper, the autonomous landing control problem on moving shipboard of the disturbed 6-DOF helicopter is studied by stabilizing the error system between the shipboard and the helicopter. The landing process is composed of two phases, i.e., homing phase, at which the helicopter is controlled to be hovered synchronously at a certain altitude over the shipboard from a distant location, and landing phase, at which the helicopter should land vertically on the shipboard in synchronization with its attitudes. Each phase is achieved by applying a composite control scheme based on a joint state and disturbance observer and a continuous TSMC (CTSMC) method. Rigorous stability analysis proves the stability of the closed-loop system. The main contributions of this paper are threefold.

1) Firstly, the landing control problem is investigated by stabilizing the error system between the shipboard and the helicopter, and meanwhile, the velocity and angular velocity of the shipboard are estimated along with lump disturbances through joint state and disturbance observers. Compared with $[14,15]$ where the motion dynamics of the shipboard is established, the proposed scheme not only achieves autonomous landing on the premise of avoiding the complicated shipboard modeling process, but also avoids the errors caused by the unmodeled shipboard dynamics and state measurements.

2) Secondly, by assembling the observer and CTSMC method, composite control schemes are proposed to achieve autonomous landing. In such case, the lump disturbances are compensated rapidly by the observer and further supressed by CTSMC method in a robust way, which significantly improves the anti-disturbance performance of the closed-loop system.

3) Thirdly, a novel dynamic terminal sliding manifold is selected and then a CTSMC method is proposed. In contrast to the literature with traditional SMC method [14], the proposed CTSMC method based scheme significantly mitigates the chattering phenomenon as well as simplifying the controller design process.

The paper is organized as follows. Some preliminaries are given in Section 2. In Section 3, the 6-DOF rigid helicopter model and control objective are presented. The landing control schemes are derived in Section 4. Section 5 introduces the numerical simulations. Finally, conclusions are provided in Section 6.

\section{Preliminaries}

\subsection{Mathematical notation}

The abbreviations $S_{t}, C_{t}$, and $T_{t}$ with $t \in \mathbb{R}$ represent the trigonometric functions $\sin (t), \cos (t)$, and $\tan (t)$, respectively. The notation " $\times$ " represents the cross product of two vectors.

For $x=\left[x_{1}, \ldots, x_{n}\right]^{T} \in \mathbb{R}^{n}, \alpha=\left[\alpha_{1}, \ldots, \alpha_{n}\right]^{T} \in \mathbb{R}^{n}$, and $\beta \in \mathbb{R}, \hat{x}=\left[\hat{x}_{1}, \ldots, \hat{x}_{n}\right]^{T}, \dot{x}=\left[\dot{x}_{1}, \ldots, \dot{x}_{n}\right]^{T}$, and $\operatorname{sig}^{\alpha}(x)=\left[\operatorname{sig}^{\alpha_{1}}\left(x_{1}\right), \ldots, \operatorname{sig}^{\alpha_{n}}\left(x_{n}\right)\right]^{T}, \operatorname{sig}^{\beta}(x)=\left[\operatorname{sig}^{\beta}\left(x_{1}\right), \ldots, \operatorname{sig}^{\beta}\left(x_{n}\right)\right]^{T}$, where $\operatorname{sig}^{b}(z)=|z|^{b} \operatorname{sgn}(z), \forall b, z \in$ $\mathbb{R}$ and $\operatorname{sgn}(z)$ is the standard sign function, namely, $\operatorname{sgn}(z)=1$ for $z>0, \operatorname{sgn}(z)=0$ for $z=0$, and $\operatorname{sgn}(\mathrm{z})=-1$ for $z<0$. Especially, $\operatorname{sgn}(x)=\left[\operatorname{sgn}\left(x_{1}\right), \ldots, \operatorname{sgn}\left(x_{n}\right)\right]^{T}$.

Given a matrix $A_{m \times n} \in \mathbb{R}^{m \times n}, \dot{A}_{m \times n} \in \mathbb{R}^{m \times n}$ represents the time derivative of matrix $A$ where each element is the time derivative of the corresponding element in $A$. 


\subsection{Lemmas}

To develop the controller, the following lemmas are given.

Lemma 1 [32] Consider the system

$$
\dot{x}=f(t, x, u),
$$

if the unforced system $\dot{x}=f(t, x, 0)$ has globally exponentially stable equilibrium point at the origin $x=0$, then the system (1) is input-to-state stable (ISS).

Lemma 2 [32] If the system (1) is ISS and $\lim _{t \rightarrow \infty} u(t)=0$, then the system state will asymptotically converge to zero, that is, $\lim _{t \rightarrow \infty} x(t)=0$.

Lemma 3 [33] If $q$ is a real number satisfying $0<q \leq 1$, then the inequality $\left(\left|x_{1}\right|+\cdots+\left|x_{n}\right|\right)^{q} \leq$ $\left|x_{1}\right|^{q}+\cdots+\left|x_{n}\right|^{q}$ holds for $x_{i} \in R, i=1, \ldots, n$.

Lemma 4 [34] Consider the system

$$
\dot{x}=f(x), \quad x \in \mathscr{U} \subseteq \mathbb{R}^{n}, \quad f(0)=0
$$

where $f: \mathscr{U} \rightarrow \mathbb{R}^{n}$ is continuous with respect to $x$ on an open neighborhood $\mathscr{U} \subseteq \mathbb{R}^{n}$ of the origin. Assume that there exists a continuously differentiable function $V(x): \mathscr{U} \rightarrow R$ such that the following conditions hold

(1) $V(x)$ is positive definite.

(2) There exist real numbers $c>0$ and $\alpha \in(0,1)$, and an open neighborhood $\mathscr{U}_{0} \subset \mathscr{U}$ of the origin such that $\dot{V}(X)+c V^{\alpha}(x) \leq 0, x \in \mathscr{U}_{0} \backslash\{0\}$.

Then, for any given initial condition $V\left(x_{0}\right)$, system (2) is finite-time stable.

Lemma 5 [35] For the second-order system $\ddot{x}=u, x \in \mathbb{R}$, it is globally finite-time convergent under the controller

$$
u=-k_{1}|x|^{\alpha_{1}} \operatorname{sgn}(x)-k_{2}|\dot{x}|^{\alpha_{2}} \operatorname{sgn}(\dot{x}),
$$

where $k_{1}, k_{2}$ are positive constants, $\alpha_{1} \in(0,1)$, and $\alpha_{2}=\frac{2 \alpha_{1}}{1+\alpha_{1}}$.

\section{Problem formulation}

\subsection{Reference frames}

To establish the model of the helicopter, two reference frames are defined. The first one is the inertial frame defined as $\mathscr{I}=\left\{O_{e}, X_{e}, Y_{e}, Z_{e}\right\}$, where $O_{e}$ is arbitrarily fixed to a point on the earth's surface, $X_{e}$ points toward the geodetic north, $Y_{e}$ points toward the east, and $Z_{e}$ points vertically down along the gravity vector. The second is the body-fixed frame defined as $\mathscr{B}=\left\{O_{b}, X_{b}, Y_{b}, Z_{b}\right\}$, where the origin $O_{b}$ is located at the gravity center of the helicopter, $X_{b}$ points forward of the helicopter, $Y_{b}$ points to the right side of the helicopter, and $Z_{b}$ points downward to comply with the right-hand rule. Two reference frames are illustrated in Fig. 1. 


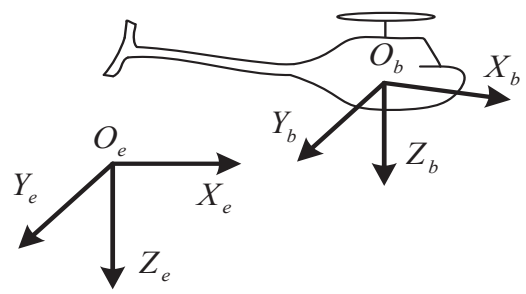

Fig. 1 Helicopter frames

\subsection{Modeling of the 6-DOF rigid helicopter}

Unmanned helicopters are strongly nonlinear systems with complex dynamic couplings, and they are easily affected by complex external disturbances, especially during autonomous shipboard landing missions. To establish a helicopter model which not only reflects the nonlinear dynamics roundly but also facilitates the controller design, a general method is to establish a simplified 6-DOF rigid-body model by treating other trivial factors affecting the helicopter as uncertainties or disturbances (which are seen as lump disturbances in this paper).

According to [36], the 6-DOF rigid-body model of the unmanned helicopter is given by

$$
\left\{\begin{array}{l}
\dot{P}=V, \\
\dot{V}=g e_{3}+\frac{1}{m} R(\Theta)\left(F+F_{d}\right), \\
\dot{\Theta}=H(\Theta) \Omega, \\
\dot{\Omega}=-J^{-1} \Omega \times J \Omega+J^{-1}\left(\Gamma+\Gamma_{d}\right),
\end{array}\right.
$$

where $P=[x, y, z]^{T}$ and $V=[u, v, w]^{T}$ represent the positions and velocities of the helicopter in inertial frame $\mathscr{I}$, respectively. $\Theta=[\phi, \theta, \psi]^{T}$ and $\Omega=[p, q, r]^{T}$ denote, respectively, the Euler attitude angles (i.e., roll angle, pitch angle, and yaw angle) and angular velocities of the helicopter in body-fixed frame $\mathscr{B}$. $g$ is the acceleration of gravity, $e_{3}=[0,0,1]^{T}, m$ is the mass of the helicopter, and $J=\operatorname{diag}\left\{J_{x x}, J_{y y}, J_{z z}\right\}$ is the diagonal inertia matrix. $R(\Theta)$ stands for the rotation matrix from body-fixed frame $\mathscr{B}$ to inertia frame $\mathscr{I}$, which is defined as

$$
R(\Theta)=\left[\begin{array}{ccc}
C_{\theta} C_{\psi} & S_{\phi} S_{\theta} C_{\psi}-C_{\phi} S_{\psi} & C_{\phi} S_{\theta} C_{\psi}+S_{\phi} S_{\psi} \\
C_{\theta} S_{\psi} & S_{\phi} S_{\theta} S_{\psi}+C_{\phi} C_{\psi} & C_{\phi} S_{\theta} S_{\psi}-S_{\phi} C_{\psi} \\
-S_{\theta} & S_{\phi} C_{\theta} & C_{\phi} C_{\theta}
\end{array}\right]
$$

The attitude kinematic matrix $H(\Theta)$ is given by

$$
H(\Theta)=\left[\begin{array}{ccc}
1 & S_{\phi} T_{\theta} & C_{\phi} T_{\theta} \\
0 & C_{\phi} & -S_{\phi} \\
0 & S_{\phi} / C_{\theta} & C_{\phi} / C_{\theta}
\end{array}\right]
$$

$F_{d}$ and $\Gamma_{d}$ represent the lumped force and torque disturbances. $F$ and $\Gamma$ represent the force and torque in body-fixed frame $\mathscr{B}$.

In translational dynamics, it is konwn that the primary force is the thrust of the main rotor along $Z_{b}$ axis. Hence, $F$ is expressed as

$$
F=[0,0, T]^{T}, T=m\left(-g+Z_{w} w+Z_{c o l} \delta_{c o l}\right),
$$

where $\delta_{c o l}$ represents the collective pitch of main rotor, $Z_{w}$ and $Z_{c o l}$ are system parameters. 
In rotation dynamics, $\Gamma$ is described as

$$
\Gamma=J\left[\begin{array}{c}
L_{a} a+L_{b} b \\
M_{a} a+M_{b} b \\
N_{r} r+N_{c o l} \delta_{c o l}+N_{p e d} \delta_{p e d}
\end{array}\right]
$$

where $L_{a}, L_{b}, M_{a}, M_{b}, N_{r}, N_{c o l}$, and $N_{p e d}$ are system parameters, whose values can be obtained by system identification. $\delta_{p e d}$ is the collective pitch of tail rotor. $a$ and $b$ represent, respectively, the flapping angles of the main rotor along the longitudinal and lateral axes, which are originally driven by the lateral cyclic $\delta_{l a t}$ and the longitudinal cyclic $\delta_{l o n}$. In steady state, the relationship between flapping angles $a, b$ and control inputs $\delta_{\text {lat }}, \delta_{\text {lon }}$ is approximated by [37]

$$
\left\{\begin{array}{l}
a=-\tau_{m} q+A_{\text {lon }} \delta_{\text {lon }}+A_{\text {lat }} \delta_{\text {lat }}, \\
b=-\tau_{m} p+B_{\text {lon }} \delta_{\text {lon }}+B_{\text {lat }} \delta_{\text {lat }}
\end{array}\right.
$$

where $\tau_{m}$ is the main rotor's dynamics time constant, $A_{\text {lon }}, A_{\text {lat }}, B_{\text {lon }}$, and $B_{\text {lat }}$ are system parameters.

Combining (3)-(6) together, the simplified 6-DOF rigid-body model of the helicopter is expressed as

$$
\left\{\begin{array}{l}
\dot{P}=V, \\
\dot{V}=g e_{3}+R(\Theta) e_{3}\left(-g+Z_{w} w+Z_{c o l} \delta_{c o l}\right)+d_{V}, \\
\dot{\Theta}=H(\Theta) \Omega \\
\dot{\Omega}=-J^{-1} \Omega \times J \Omega+A \Omega+B U+d_{\Omega},
\end{array}\right.
$$

where $U=\left[\delta_{c o l}, \delta_{l o n}, \delta_{l a t}, \delta_{p e d}\right]^{T}$ denotes the control input vector, $A=\left[\begin{array}{ccc}-\tau_{m} L_{b} & -\tau_{m} L_{a} & 0 \\ -\tau_{m} M_{b} & -\tau_{m} M_{a} & 0 \\ 0 & 0 & N_{r}\end{array}\right]$ and $B=$ $\left[\begin{array}{cccc}0 & L_{\text {lon }} & L_{\text {lat }} & 0 \\ 0 & M_{\text {lon }} & M_{\text {lat }} & 0 \\ N_{\text {col }} & 0 & 0 & N_{\text {ped }}\end{array}\right]$, where $L_{\text {lat }}=L_{a} A_{\text {lat }}+L_{b} B_{\text {lat }}, L_{\text {lon }}=L_{a} A_{\text {lon }}+L_{b} B_{\text {lon }}, M_{\text {lat }}=M_{a} A_{\text {lat }}+M_{b} B_{\text {lat }}$, and $M_{l o n}=M_{a} A_{l o n}+M_{b} B_{l o n} . d_{V}=\frac{1}{m} R(\Theta) F_{d}$ and $d_{\Omega}=J^{-1} \Gamma_{d}$ represent lumped disturbances acting on the helicopter.

Remark 1 This paper deals with smooth landing maneuver of the helicopter. Hence, the roll and pitch angles of the helicopter system (7) are considered to satisfy $|\phi(t)|<\frac{\pi}{2}$ and $|\theta(t)|<\frac{\pi}{2}$ for $t \geq 0$. In this case, the kinematic matrix $H(\Theta)$ is nonsingular. It is reasonable because with appropriate control strategies, the roll and pitch angles of the helicopter can be guaranteed to be within the ranges of $\pm \frac{\pi}{2}$ during normal landing process. Similar statements are also made in $[12,15]$ and the references therein.

\subsection{Control objective}

The objective of this paper is to design the controller $U=\left[\delta_{c o l}, \delta_{l o n}, \delta_{l a t}, \delta_{p e d}\right]^{T}$ for the unmanned helicopter system (7) such that it lands on the moving shipboard in the presence of lump disturbances. The landing process consists of two phases, i.e., homing phase and landing phase. For homing phase, the homing controller $U$ is designed such that the positions $P=[x, y, z]^{T}$ and yaw angle $\psi$ of the helicopter converge to the desired position $P_{d}=\left[x_{s}, y_{s}, z_{d}\right]^{T}$ and yaw angle $\psi_{d}$ asymptotically, where $x_{s}, y_{s}$ are actual positions of the shipboard, $z_{d}$ and $\psi_{d}$ are predefined values. For landing phase, the landing controller $U$ is designed to make the attitude angles $\Theta=[\phi, \theta, \psi]^{T}$ and vertical position $z$ of the helicopter converge to the attitude angles $\Theta_{s}=\left[\phi_{s}, \theta_{s}, \psi_{s}\right]^{T}$ and vertical position $z_{s}$ of the shipboard in finite time, where $\phi_{s}, \theta_{s}, \psi_{s}$ denote three Euler attitude angles of the shipboard. The landing process is presented in Fig. 2. 


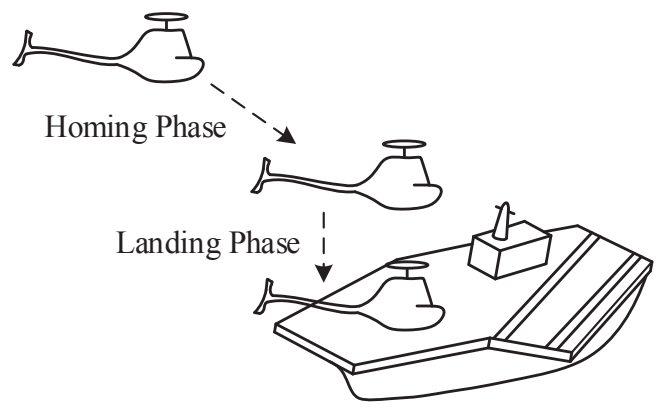

Fig. 2 Landing process: homing phase and landing phase

\section{Controller design}

Before proceeding, an assumption is made.

Assumption 1 All states of the helicopter are measurable. The positions and attitude angles of the shipboard are measurable.

\subsection{Homing phase}

In homing phase, the goal is to design the homing controller $U$ such that the helicopter is hovered synchronously at the predefined altitude $z_{d}$ over the shipboard from a distant location. To design the controller, a hierarchical double-loop control scheme is proposed where the slower dynamics (translational dynamics) is the outer-loop and the faster dynamics (rotational dynamics) is the inner-loop [15,21]. Since the rotational dynamics is much faster than the translational dynamics, it is considered that the virtual desired signals $\phi_{d}, \theta_{d}$ generated from the translational dynamics have been obtained from the rotational dynamics at any sample time. Therefore, a relative velocity and disturbance observer (RVDO) is integrated with the relative position feedback control to achieve asymptotic control in outer-loop while a HOSMO and the CTSMC method are combined to achieve finite-time control in inner-loop.

\subsubsection{Translational subsystem}

Take $P_{d}=\left[x_{s}, y_{s}, z_{d}\right]^{T}$ as the desired position. Define $e_{1}=\left[e_{1, x}, e_{1, y}, e_{1, z}\right]^{T}=P-P_{d}$ as the position tracking error, and $e_{2}=\left[e_{2, x}, e_{2, y}, e_{2, z}\right]^{T}=V-\dot{P}_{d}$. By invoking (7), the translational tracking error subsystem is obtained

$$
\left\{\begin{array}{l}
\dot{e}_{1}=e_{2}, \\
\dot{e}_{2}=U_{1}+d_{1},
\end{array}\right.
$$

where $U_{1}=\left[U_{1, x}, U_{1, y}, U_{1, z}\right]^{T}=g e_{3}+R(\Theta) e_{3}\left(-g+Z_{w} w+Z_{c o l} \delta_{c o l}\right)$, and $d_{1}=\left[d_{1, x}, d_{1, y}, d_{1, z}\right]^{T}=d_{V}-\ddot{P}_{d}$.

From Assumption 1, it is obtained that the first-order and second-order derivatives of $x_{s}$ and $y_{s}$ are unknown, namely, $\dot{P}_{d}$ and $\ddot{P}_{d}$ are unknown. Hence, $e_{2}$ and $d_{1}$ in (8) are unknown. To this end, a RVDO is designed to estimate $e_{2}$ and $d_{1}$ together. The estimates are then incorporated into the relative position feedback control such that a composite position tracking scheme is proposed for the position tracking controller $U_{1}$. The detailed design process includes two steps, i.e., RVDO design and composite position tracking controller design.

Assumption 2 The lump disturbances $d_{1, i}, i \in\{x, y, z\}$ in (8) are regarded as the combination of $m_{i}$ harmonic components $x_{1, i}, x_{3, i}, \ldots, x_{2 m_{i}-1, i}$ with different frequencies $\omega_{1, i}, \omega_{3, i}, \ldots, \omega_{2 m_{i}-1, i}$, respectively, and one polynomial component $D_{i}$ satisfying $\lim _{t \rightarrow \infty} D_{i}^{\left(n_{i}\right)}(t)=0$, namely, $d_{1, i}=x_{1, i}+x_{3, i}+\ldots+x_{2 m_{i}-1, i}+D_{i}$. 
Remark 2 Assumption 2 is given based on the motion model of the shipboard in [14, 15] and the construction of disturbance $d_{V}$. This description fully covers a general class of disturbances, such as constant, ramp, parabolic, higher-order, sinusoidal disturbances and the arbitrary combination of them.

\section{RVDO design}

With Assumption 2 in mind, the translational tracking error subsystem (8) is redescribed as

$$
\left\{\begin{array}{l}
\dot{e}_{1, i}=e_{2, i} \\
\dot{e}_{2, i}=U_{1, i}+x_{1, i}+x_{3, i}+\ldots+x_{2 m_{i}-1, i}+D_{i} \\
\dot{x}_{1, i}=x_{2, i} \\
\dot{x}_{2, i}=-\omega_{1, i}^{2} x_{1, i} \\
\ldots \\
\dot{x}_{2 m_{i}-1, i}=x_{2 m_{i}, i} \\
\dot{x}_{2 m_{i}, i}=-\omega_{2 m_{i}-1, i}^{2} x_{2 m_{i}-1, i}
\end{array}\right.
$$

where $i \in\{x, y, z\}, \lim _{t \rightarrow \infty} D_{i}^{\left(n_{i}\right)}(t)=0$, and $m_{i}, n_{i} \in \mathbb{N}$.

Next, a RVDO is designed to estimate $e_{2, i}$ and the disturbance components $x_{1, i}, x_{1, i}, \ldots, x_{2 m_{i}-1, i}, D_{i}$ in (9)

$$
\left\{\begin{aligned}
& \dot{z}_{0, i}=-a_{0, i}\left(z_{0, i}+a_{0, i} e_{1, i}\right)+\left(z_{1, i}+a_{1, i} e_{1, i}\right)+\left(z_{3, i}+a_{3, i} e_{1, i}\right) \\
&+\ldots+\left(z_{2 m_{i}-1, i}+a_{2 m_{i}-1, i} e_{1, i}\right)+\left(\delta_{1, i}+b_{1, i} e_{1, i}\right)+U_{1, i}, \\
& \dot{z}_{1, i}=-a_{1, i}\left(z_{0, i}+a_{0, i} e_{1, i}\right)+z_{2, i}+a_{2, i} e_{1, i}, \\
& \dot{z}_{2, i}=-a_{2, i}\left(z_{0, i}+a_{0, i} e_{1, i}\right)-\omega_{1, i}^{2}\left(z_{1, i}+a_{1, i} e_{1, i}\right), \\
& \ldots \\
& \dot{z}_{2 m_{i}-1, i}=-a_{2 m_{i}-1, i}\left(z_{0, i}+a_{0, i} e_{1, i}\right)+z_{2 m_{i}, i}+a_{2 m_{i}, i} e_{1, i}, \\
& \dot{z}_{2 m_{i}, i}=-a_{2 m_{i}, i}\left(z_{0, i}+a_{0, i} e_{1, i}\right)-\omega_{2 m_{i}-1, i}^{2}\left(z_{2 m_{i}-1, i}+a_{2 m_{i}-1, i} e_{1, i}\right), \\
& \dot{\delta}_{1, i}=-b_{1, i}\left(z_{0, i}+a_{0, i} e_{1, i}\right)+\delta_{2, i}+b_{2, i} e_{1, i}, \\
& \ldots \\
& \dot{\delta}_{n_{i}-1, i}=-b_{n_{i}-1, i}\left(z_{0, i}+a_{0, i} e_{1, i}\right)+\delta_{n_{i}, i}+b_{n_{i}, i} e_{1, i}, \\
& \dot{\delta}_{n_{i}, i}=-b_{n_{i}, i}\left(z_{0, i}+a_{0, i} e_{1, i}\right),
\end{aligned}\right.
$$

where $i \in\{x, y, z\}, a_{0, i}, a_{1, i}, \ldots, a_{2 m_{i}, i}, b_{1, i}, \ldots, b_{n_{i}, i}$ are observer gains to be assigned later. $\hat{e}_{2, i}=z_{0, i}+$ $a_{0, i} e_{1, i}, \hat{x}_{1, i}=z_{1, i}+a_{1, i} e_{1, i}, \hat{x}_{2, i}=z_{2, i}+a_{2, i} e_{1, i}, \ldots, \hat{x}_{2 m_{i}, i}=z_{2 m_{i}, i}+a_{2 m_{i}, i} e_{1, i}, \hat{D}_{i}=\delta_{1, i}+b_{1, i} e_{1, i}, \ldots$, $\widehat{D_{i}^{\left(n_{i}-1\right)}}=\delta_{n_{i}, i}+b_{n_{i}, i} e_{1, i}$, where $\hat{e}_{2, i}, \hat{x}_{1, i}, \hat{x}_{2, i}, \ldots, \hat{x}_{2 m_{i}, i}, \hat{D}_{i}, \ldots$, and $\widehat{D_{i}^{\left(n_{i}-1\right)}}$ are the estimates of $e_{2, i}, x_{1, i}$, $x_{2, i}, \ldots, x_{2 m_{i}, i}, D_{i}, \ldots$, and $D_{i}^{\left(n_{i}-1\right)}$, respectively.

Define the estimation errors as $\varepsilon_{0, i}=\hat{e}_{2, i}-e_{2, i}, \varepsilon_{1, i}=\hat{x}_{1, i}-x_{1, i}, \varepsilon_{2, i}=\hat{x}_{2, i}-x_{2, i}, \ldots, \varepsilon_{2 m_{i}, i}=\hat{x}_{2 m_{i}, i}-$ $x_{2 m_{i}, i}, \sigma_{1, i}=\hat{D}_{i}-D_{i}, \ldots, \sigma_{n_{i}, i}=\widehat{D_{i}^{\left(n_{i}-1\right)}}-D_{i}^{\left(n_{i}-1\right)}$. Taking the time derivatives of estimation errors, the estimation error dynamics is obtained

$$
\dot{\Lambda}_{i}=\Phi_{i} \Lambda_{i}+\Pi_{i},
$$


where $\Lambda_{i}=\left[\varepsilon_{0, i}, \varepsilon_{1, i}, \varepsilon_{2, i}, \ldots, \varepsilon_{2 m_{i}, i}, \sigma_{1, i}, \ldots, \sigma_{n_{i}, i}\right]^{T} \in \mathbb{R}^{2 m_{i}+n_{i}+1}, \Pi_{i}=\left[0,0, \ldots,-D_{i}^{\left(n_{i}\right)}\right]^{T} \in \mathbb{R}^{2 m_{i}+n_{i}+1}$,

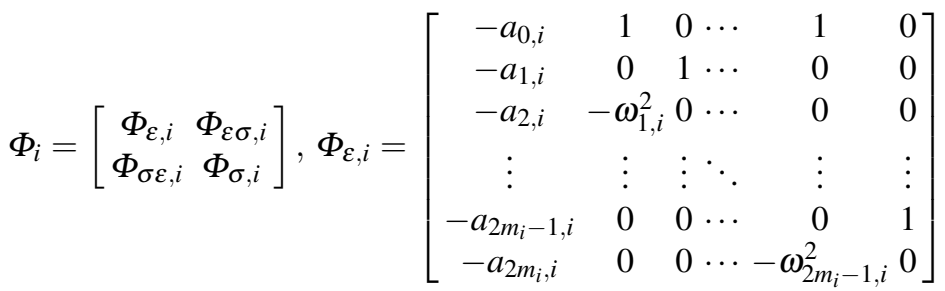

$$
\in \mathbb{R}^{\left(2 m_{i}+1\right) \times\left(2 m_{i}+1\right)}, \Phi_{\varepsilon \sigma, i}=
$$

$\left[\begin{array}{cc}1 & 0_{1 \times\left(n_{i}-1\right)} \\ 0_{2 m_{i} \times 1} & 0_{2 m_{i} \times\left(n_{i}-1\right)}\end{array}\right] \in \mathbb{R}^{\left(2 m_{i}+1\right) \times n_{i}}, \Phi_{\sigma \varepsilon, i}=\left[\begin{array}{cccc}-b_{1, i} & 0 & \cdots & 0 \\ \vdots & \vdots & \ddots & \vdots \\ -b_{n_{i}-1, i} & 0 & \cdots & 0 \\ -b_{n_{i}, i} & 0 & \cdots & 0\end{array}\right] \in \mathbb{R}^{n_{i} \times\left(2 m_{i}+1\right)}, \Phi_{\sigma, i}=\left[\begin{array}{cc}0_{\left(n_{i}-1\right) \times 1} & I_{n_{i}-1} \\ 0 & 0_{1 \times\left(n_{i}-1\right)}\end{array}\right] \in$

$\mathbb{R}^{n_{i} \times n_{i}}$

Proposition 1 The estimation error of the designed RVDO (10) asymptotically converge to zero, namely, $\lim _{t \rightarrow \infty} \Lambda_{i}(t)=0, i \in\{x, y, z\}$, provided that the observer gains $a_{0, i}, a_{1, i}, \ldots, a_{2 m_{i}, i}, b_{1, i}, \ldots, b_{n_{i}, i}$ are selected such that $\Phi_{i}$ in (11) is a Hurwitz matrix.

Proof For a certain $i \in\{x, y, z\}$, the reduced system $\dot{\Lambda}_{i}=\Phi_{i} \Lambda_{i}$ of (11) is globally exponentially stable if $\Phi_{i}$ is a Hurwitz matrix. Regarding $\Pi_{i}$ as the input of (11), then it follows from Lemma 1 that the estimation error dynamics (11) is ISS. Note that $\lim _{t \rightarrow \infty} D_{i}^{\left(n_{i}\right)}=0$, namely, $\lim _{t \rightarrow \infty} \Pi_{i}=0$. Then it can be deduced from Lemma 2 that the state of (11) asymptotically converge to zero, that is, $\lim _{t \rightarrow \infty} \Lambda_{i}(t)=0$. This completes the proof.

\section{Composite position tracking controller design}

Design the position tracking controller $U_{1}$ as

$$
U_{1}=-K_{1} e_{1}-K_{2} \hat{e}_{2}-\hat{d}_{1},
$$

where $K_{1}=\operatorname{diag}\left\{k_{1, x}, k_{1, y}, k_{1, z}\right\}$ and $K_{2}=\operatorname{diag}\left\{k_{2, x}, k_{2, y}, k_{2, z}\right\}$ are positive definite diagonal matrices, $\hat{d}_{1}=$ $\left[\hat{d}_{1, x}, \hat{d}_{1, y}, \hat{d}_{1, z}\right]^{T}, \hat{d}_{1, i}=\hat{x}_{1, i}+\hat{x}_{3, i}+\ldots+\hat{x}_{2 m_{i}-1, i}+\hat{D}_{i}, i \in\{x, y, z\}$.

Theorem 1 Consider the translational tracking error subsystem (8) under the controller (12) with the $R V D O$ (10). If the observer gains $a_{0, i}, a_{1, i}, \ldots, a_{2 m_{i}, i}, b_{1, i}, \ldots, b_{n_{i}, i}, i \in\{x, y, z\}$ in (10) are selected such that $\Phi_{i}$ in (11) is a Hurwitz matrix, and the controller gains $k_{1, i}, k_{2, i}$ in (12) are selected such that $C_{i}$ in (13) is a Hurwitz matrix, then the closed-loop translational tracking error subsystem (8) and (12) is asymptotically stable, that is, the position tracking error $e_{1}$ converges to zero asymptotically.

Proof Define $E_{i}=\left[e_{1, i}, e_{2, i}\right]^{T}, i \in\{x, y, z\}$. Substituting (12) into (9), the translational tracking error subsystem is then governed by

$$
\dot{E}_{i}=C_{i} E_{i}+h_{i}
$$

where $h_{i}=\left[0,-\left(k_{2, i} \varepsilon_{0, i}+\varepsilon_{1, i}+\varepsilon_{3, i}+\ldots+\varepsilon_{2 m_{i}-1, i}+\sigma_{1, i}\right)\right]^{T}, C_{i}=\left[\begin{array}{cc}0 & 1 \\ -k_{1, i}-k_{2, i}\end{array}\right]$. Taking $h_{i}$ as the input of (13), the reduced system $\dot{E}_{i}=C_{i} E_{i}$ is globally exponentially stable if $C_{i}$ is chosen as a Hurwitz matrix. Then from Lemma 1, the translational tracking error subsystem (13) is ISS. Based on Proposition 1 , if $\Phi_{i}$ in (11) is a Hurwitz matrix, then $\lim _{t \rightarrow \infty} \Lambda_{i}=0$, namely, $\lim _{t \rightarrow \infty} h_{i}=0$. Combining this with Lemma 2 together, it gives that the translational tracking error subsystem (13) is asymptotically stable, that is, $\lim _{t \rightarrow \infty} e_{1}(t)=0$. This completes the proof. 
By the definition of $U_{1}, \delta_{c o l}$ and the virtual desired angles are given by

$$
\begin{gathered}
\delta_{c o l}=\frac{\sqrt{U_{1, x}^{2}+U_{1, y}^{2}+\left(U_{1, z}-g\right)^{2}}+g-Z_{w} w}{Z_{c o l}} . \\
\left\{\begin{array}{l}
\phi_{d}=\arcsin \frac{U_{1, x} S_{\psi}-U_{1, y} C_{\psi}}{\sqrt{U_{1, x}^{2}+U_{1, y}^{2}+\left(U_{1, z}-g\right)^{2}}} \\
\theta_{d}=\arctan \frac{U_{1, x} C_{\psi}+U_{1, y} S_{\psi}}{U_{1, z}-g} .
\end{array}\right.
\end{gathered}
$$

\subsubsection{Rotational subsystem}

Take $\Theta_{d}=\left[\phi_{d}, \theta_{d}, \psi_{d}\right]^{T}$, where $\phi_{d}$ and $\theta_{d}$ are virtual desired pitch and roll angles derived from (15), $\psi_{d}$ is the desired yaw angle. Define $e_{3}=\left[e_{3, \phi}, e_{3, \theta}, e_{3, \psi}\right]^{T}=\Theta-\Theta_{d}$ as the attitude tracking error, and $e_{4}=\left[e_{4, \phi}, e_{4, \theta}, e_{4, \psi}\right]^{T}=H \Omega-\dot{\Theta}_{d}$. The rotational tracking error subsystem is then given by

$$
\left\{\begin{array}{l}
\dot{e}_{3}=e_{4} \\
\dot{e}_{4}=f_{1}+U_{2}+d_{2}
\end{array}\right.
$$

where $f_{1}=\left[f_{1, \phi}, f_{1, \theta}, f_{1, \psi}\right]^{T}=\dot{H} \Omega+H\left(-J^{-1} \Omega \times J \Omega+A \Omega\right)-\ddot{\Theta}_{d}, U_{2}=\left[U_{2, \phi}, U_{2, \theta}, U_{2, \psi}\right]^{T}=H B U$, and $d_{2}=\left[d_{2, \phi}, d_{2, \theta}, d_{2, \psi}\right]^{T}=H d_{\Omega}$.

Next, a composite attitude tracking scheme is proposed for the attitude tracking controller $U_{2}$ by combining HOSMO and the CTSMC method. The detailed design process consists of two steps, i.e., HOSMO design and composite attitude tracking controller design.

Assumption 3 For the rotational tracking error subsystem (16), the lump disturbances $d_{2, i}, i \in\{\phi, \theta, \psi\}$ satisfy $\left|\dot{d}_{2, i}\right| \leq L_{i}$, where $L_{i}$ are known constants.

\section{HOSMO design}

The following HOSMO is designed to estimate $d_{2}$ in (16)

$$
\left\{\begin{array}{l}
\dot{\varpi}_{1}=f_{1}+U_{2}+v_{1}, v_{1}=-\lambda_{1} L^{\frac{1}{2}} \operatorname{sig}^{\frac{1}{2}}\left(\varpi_{1}-e_{4}\right)+\varpi_{2}, \\
\dot{\varpi}_{2}=-\lambda_{2} L \operatorname{sgn}\left(\varpi_{2}-v_{1}\right)
\end{array}\right.
$$

where $\varpi_{1}$ and $\varpi_{2}$ are the estimates of $e_{4}$ and $d_{2}$, respectively. $L=\operatorname{diag}\left\{L_{\phi}, L_{\theta}, L_{\psi}\right\}, \lambda_{1}, \lambda_{2} \in \mathbb{R}^{3 \times 3}$ are observer gains to be determined, which are both positive definite diagonal matrices.

Define the estimation errors as $\xi_{1}=\left[\xi_{1, \phi}, \xi_{1, \theta}, \xi_{1, \psi}\right]^{T}=\varpi_{1}-e_{4}$ and $\xi_{2}=\left[\xi_{2, \phi}, \xi_{2, \theta}, \xi_{2, \psi}\right]^{T}=\varpi_{2}-d_{2}$. Then the estimation error dynamics is given by

$$
\left\{\begin{array}{l}
\dot{\xi}_{1}=-\lambda_{1} L^{\frac{1}{2}} \operatorname{sig}^{\frac{1}{2}}\left(\xi_{1}\right)+\xi_{2}, \\
\dot{\xi}_{2}=-\lambda_{2} L \operatorname{sgn}\left(\xi_{2}-\dot{\xi}_{1}\right)-\dot{d}_{2} .
\end{array}\right.
$$

From [28, 29], it is proved that the error dynamics (18) is finite-time stable provided that the observer scaler gains $L_{i} \geq\left|\dot{d}_{2, i}\right|, i \in\{\phi, \theta, \psi\}$. This means that all signals in (18) are bounded and there exists a finite time constant $T_{r}>0$ such that $\xi_{1} \equiv 0, \xi_{2} \equiv 0$, for $t \geq T_{r}$.

II. Composite attitude tracking controller design

A novel dynamic terminal sliding manifold $s_{1}$ is designed as

$$
s_{1}=K_{3} \operatorname{sig}^{\alpha_{1}}\left(e_{3}\right)+K_{4} \operatorname{sig}^{\alpha_{2}}\left(e_{4}\right)+f_{1}+U_{2}+\hat{d}_{2},
$$


where $s_{1}=\left[s_{1, \phi}, s_{1, \theta}, s_{1, \psi}\right]^{T} . K_{3}=\operatorname{diag}\left\{k_{3, \phi}, k_{3, \theta}, k_{3, \psi}\right\}$ and $K_{4}=\operatorname{diag}\left\{k_{4, \phi}, k_{4, \theta}, k_{4, \psi}\right\}$ are positive definite diagonal matrices. $\alpha_{1}=\left[\alpha_{1, \phi}, \alpha_{1, \theta}, \alpha_{1, \psi}\right]^{T}, \alpha_{2}=\left[\alpha_{2, \phi}, \alpha_{2, \theta}, \alpha_{2, \psi}\right]^{T}$, and $0<\alpha_{1, i}<1, \alpha_{2, i}=\frac{2 \alpha_{1, i}}{1+\alpha_{1, i}}, i \in$ $\{\phi, \theta, \psi\} . \hat{d}_{2}=\left[\hat{d}_{2, \phi}, \hat{d}_{2, \theta}, \hat{d}_{2, \psi}\right]^{T}$ represents the estimates of $d_{2}$, namely, $\hat{d}_{2}=\varpi_{2}$.

Design the attitude tracking controller $U_{2}$ as

$$
\left\{\begin{array}{l}
U_{2}=-\left(U_{2}^{e q}-U_{2}^{n}\right) \\
U_{2}^{e q}=K_{3} \operatorname{sig}^{\alpha_{1}}\left(e_{3}\right)+K_{4} \operatorname{sig}^{\alpha_{2}}\left(e_{4}\right)+f_{1}+\hat{d}_{2} \\
\dot{U}_{2}^{n}=-\eta_{1} \operatorname{sgn}\left(s_{1}\right)
\end{array}\right.
$$

where $\eta_{1}=\operatorname{diag}\left\{\eta_{1, \phi}, \eta_{1, \theta}, \eta_{1, \psi}\right\}$ is a positive definite diagonal matrix.

Theorem 2 Consider the rotational tracking error subsystem (16) under the attitude tracking controller (20) with the HOSMO (17). If the observer scaler gains $L_{i}, i \in\{\phi, \theta, \psi\}$ in (17) are chosen such that $L_{i} \geq\left|\dot{d}_{2, i}\right|$, then the closed-loop rotational tracking error subsystem (16) and (20) is finite-time convergent, that is, the attitude tracking error $e_{3}$ converges to zero in finite time.

Proof The proof consists of three steps. The first step is to prove that $e_{3}, e_{4}$ of (16) and $s_{1}$ of (19) are not escape to infinity during the period before the estimation errors of HOSMO (17) converge to zero. In the second step, it is proved that the dynamic terminal sliding manifold $s_{1}$ converges to zero in finite time. Finally, the finite-time convergence of the attitude tracking error $e_{3}$ is proved in the third step.

Step 1: When $t<T_{r}$, choose a finite-time bounded function in terms of $e_{3}, e_{4}$, and $s_{1}$ as

$$
V_{1}=\frac{1}{2}\left(e_{3}^{T} e_{3}+e_{4}^{T} e_{4}+s_{1}^{T} s_{1}\right)
$$

Taking the time derivative of $V_{1}$ yields

$$
\begin{aligned}
\dot{V}_{1}= & \left(e_{3, \phi} \dot{e}_{3, \phi}+e_{4, \phi} \dot{e}_{4, \phi}+s_{1, \phi} \dot{s}_{1, \phi}\right) \\
& +\left(e_{3, \theta} \dot{e}_{3, \theta}+e_{4, \theta} \dot{e}_{4, \theta}+s_{1, \theta} \dot{s}_{1, \theta}\right) \\
& +\left(e_{3, \psi} \dot{e}_{3, \psi}+e_{4, \psi} \dot{e}_{4, \psi}+s_{1, \psi} \dot{s}_{1, \psi}\right) .
\end{aligned}
$$

For a certain $i \in\{\phi, \theta, \psi\}$, it concluded from (16), (19), and (20) that

$$
\begin{aligned}
\dot{e}_{4, i} & =f_{1, i}+U_{2, i}+d_{2, i} \\
& =s_{1, i}-k_{3, i} \operatorname{sig}^{\alpha_{1, i}}\left(e_{3, i}\right)-k_{4, i} \operatorname{sig}^{\alpha_{2, i}}\left(e_{4, i}\right)-\hat{d}_{2, i}+d_{2, i} \\
& \leq\left|s_{1, i}\right|+k_{3, i}\left|e_{3, i}\right|^{\alpha_{1, i}}+k_{4, i}\left|e_{4, i}\right|^{\alpha_{2, i}}+\left|\xi_{2, i}\right| .
\end{aligned}
$$

Since $\alpha_{1, i}, \alpha_{2, i} \in(0,1)$, one obtains $\left|e_{3, i}\right|^{\alpha_{1, i}}<1+\left|e_{3, i}\right|,\left|e_{4, i}\right|^{\alpha_{2, i}}<1+\left|e_{4, i}\right|$. Hence, (23) is rewritten as

$$
\dot{e}_{4, i} \leq\left|s_{1, i}\right|+k_{3, i}\left(1+\left|e_{3, i}\right|\right)+k_{4, i}\left(1+\left|e_{4, i}\right|\right)+\left|\xi_{2, i}\right| .
$$


Furthermore, it can be obtained that

$$
\begin{aligned}
& e_{3, i} \dot{e}_{3, i}+e_{4, i} \dot{e}_{4, i}+s_{1, i} \dot{1}_{1, i} \\
\leq & e_{3, i} e_{4, i}+\left|e_{4, i}\right|\left|s_{1, i}\right|+\left|e_{4, i}\right|\left(k_{3, i}+k_{4, i}\right)+k_{3, i}\left|e_{4, i}\right|\left|e_{3, i}\right| \\
& +k_{4, i} e_{4, i}^{2}+\left|e_{4, i}\right|\left|\xi_{2, i}\right|-\eta_{1, i}\left|s_{1, i}\right| \\
\leq & \frac{e_{3, i}^{2}+e_{4, i}^{2}}{2}+\frac{e_{4, i}^{2}+s_{1, i}^{2}}{2}+\left(k_{3, i}+k_{4, i}+\left|\xi_{2, i}\right|\right) \frac{e_{4, i}^{2}+1}{2} \\
& +k_{3, i} \frac{e_{3, i}^{2}+e_{4, i}^{2}}{2}+k_{4, i} e_{4, i}^{2} \\
= & \left(1+k_{3, i}\right) \frac{e_{3, i}^{2}}{2}+\left(2+2 k_{3, i}+3 k_{4, i}+\left|\xi_{2, i}\right|\right) \frac{e_{4, i}^{2}}{2} \\
& +\frac{s_{1, i}^{2}}{2}+\frac{1}{2}\left(k_{3, i}+k_{4, i}+\left|\xi_{2, i}\right|\right) \\
\leq & \frac{1}{2} W_{i}\left(e_{3, i}^{2}+e_{4, i}^{2}+s_{1, i}^{2}\right)+R_{i},
\end{aligned}
$$

where $W_{i}=2+2 k_{3, i}+3 k_{4, i}+\left|\xi_{2, i}\right|$ and $R_{i}=\frac{1}{2}\left(k_{3, i}+k_{4, i}+\left|\xi_{2, i}\right|\right)$. Therefore, the time derivative of $V_{1}$ is

$$
\begin{aligned}
\dot{V}_{1} \leq & \frac{1}{2} W_{\phi}\left(e_{3, \phi}^{2}+e_{4, \phi}^{2}+s_{1, \phi}^{2}\right)+\frac{1}{2} W_{\theta}\left(e_{3, \theta}^{2}+e_{4, \theta}^{2}+s_{1, \theta}^{2}\right) \\
& +\frac{1}{2} W_{\psi}\left(e_{3, \psi}^{2}+e_{4, \psi}^{2}+s_{1, \psi}^{2}\right)+R_{\phi}+R_{\theta}+R_{\psi} \\
\leq & W_{\Theta} V_{1}+R_{\Theta},
\end{aligned}
$$

where $W_{\Theta}=\max \left\{W_{\phi}, W_{\theta}, W_{\psi}\right\}$ and $R_{\Theta}=R_{\phi}+R_{\theta}+R_{\psi}$.

The solution of (26) satisfies that

$$
V_{1}(t) \leq\left(V_{1}(0)+\frac{R_{\Theta}}{W_{\Theta}}\right) e^{W_{\Theta} t}-\frac{R_{\Theta}}{W_{\Theta}}
$$

where $V_{1}(0)$ represents the initial value of $V_{1}$. Following $\xi_{2, i}$ is bounded, it can be verified that both $W_{\Theta}$ and $R_{\Theta}$ are bounded. Hence, for any bounded time $T_{r}, V_{1}(t)$ is bounded, i.e., $e_{3}, e_{4}$, and $s_{1}$ are not escape to infinity when $t<T_{r}$.

Step 2: Construct a Lyapunov function as $V_{2}=\frac{1}{2} s_{1}^{T} s_{1}$. By substituting (20) into (19), the time derivative of $V_{2}$ is given by

$$
\begin{aligned}
\dot{V}_{2} & =-\eta_{1, \phi}\left|s_{1, \phi}\right|-\eta_{1, \theta}\left|s_{1, \theta}\right|-\eta_{1, \psi}\left|s_{1, \psi}\right| \\
& \leq-\underline{\eta_{1}}\left(\left|s_{1, \phi}\right|+\left|s_{1, \theta}\right|+\left|s_{1, \psi}\right|\right)
\end{aligned}
$$

where $\underline{\eta_{1}}=\min \left\{\eta_{1, \phi}, \eta_{1, \theta}, \eta_{1, \psi}\right\}$.

In view of Lemma 3 , the inequality $\left(s_{1, \phi}^{2}+s_{1, \theta}^{2}+s_{1, \psi}^{2}\right)^{\frac{1}{2}} \leq\left|s_{1, \phi}\right|+\left|s_{1, \theta}\right|+\left|s_{1, \psi}\right|$ holds. Following this, (28) reduces to

$$
\dot{V}_{2} \leq-\sqrt{2} \underline{\eta_{1}} V_{2}^{\frac{1}{2}}
$$

Then it can be concluded from Lemma 4 that $V_{2}$ and $s_{1}$ converge to zero in finite time, namely, there exists a finite time constant $T_{s}>0$ such that $s_{1}=0$ when $t \geq T_{s}$. 
Step 3: Substituting (19) into (16), one has

$$
\ddot{e}_{3}=-K_{3} \operatorname{sig}^{\alpha_{1}}\left(e_{3}\right)-K_{4} \operatorname{sig}^{\alpha_{2}}\left(\dot{e}_{3}\right)-\xi_{2}+s_{1} .
$$

Let $T=\max \left\{T_{r}, T_{s}\right\}$. When $t \geq T, \xi_{2}=0$ and $s_{1}=0$ are kept. Then, (30) turns to

$$
\ddot{e}_{3}=-K_{3} \operatorname{sig}^{\alpha_{1}}\left(e_{3}\right)-K_{4} \operatorname{sig}^{\alpha_{2}}\left(\dot{e}_{3}\right) \text {. }
$$

According to Lemma 5, the attitude tracking error $e_{3}$ converges to zero in finite time. This completes the proof.

With Remark 1 in mind, $H(\Theta)$ is invertible with the inverse being

$$
H^{-1}(\Theta)=\left[\begin{array}{ccc}
1 & 0 & -S_{\theta} \\
0 & C_{\phi} & S_{\phi} C_{\theta} \\
0 & -S_{\phi} & C_{\phi} C_{\theta}
\end{array}\right]
$$

By the identity $U_{2}=H B U$, it gives $H^{-1} U_{2}=B U$. Then, by some algebraic transformations, the control inputs $\delta_{\text {lon }}, \delta_{\text {lat }}$, and $\delta_{\text {ped }}$ are given as

$$
\left\{\begin{array}{l}
\delta_{l o n}=\frac{\left(C_{\phi} U_{2, \theta}+S_{\phi} C_{\theta} U_{2, \psi}\right) L_{l a t}-\left(U_{2, \phi}-S_{\theta} U_{2, \psi}\right) M_{l a t}}{L_{l a t} M_{l o n}-L_{l o n} M_{l a t}} \\
\delta_{l a t}=\frac{\left(U_{2, \phi}-S_{\theta} U_{2, \psi}\right) M_{l o n}-\left(C_{\phi} U_{2, \theta}+S_{\phi} C_{\theta} U_{2, \psi}\right) L_{l o n}}{L_{l a t} M_{l o n}-L_{l o n} M_{l a t}} \\
\delta_{\text {ped }}=\frac{-S_{\phi} U_{2, \theta}+C_{\phi} C_{\theta} U_{2, \psi}-N_{c o l} \delta_{c o l}}{N_{p e d}}
\end{array}\right.
$$

where $\delta_{c o l}$ is from (14).

According to Theorems 1 and 2, it can be concluded that under the homing controller (14) and (32), the positions $P=[x, y, z]^{T}$ and yaw angle $\psi$ of the helicopter converge to the desired position $P_{d}=\left[x_{s}, y_{s}, z_{d}\right]^{T}$ and yaw angle $\psi_{d}$ asymptotically. The hierarchical control structure for homing phase is shown in Fig. 3.

Remark 3 The attitude tracking controller $U_{2}$ is embedded in the dynamic terminal sliding manifold $s_{1}$. Part of $U_{2}$ (namely, $U_{2}^{e q}$ ) is designed to cancel out the terms other than $U_{2}$ in $s_{1}$, and the other part (namely, $\left.U_{2}^{n}\right)$ is to make $\dot{s}_{1}=-\eta_{1} \operatorname{sgn}\left(s_{1}\right)$ hold. Since $\dot{s}_{1}=\dot{U}_{2}^{n}=-\eta_{1} \operatorname{sgn}\left(s_{1}\right), U_{2}$ can be obtained directly from $s_{1}$ without actually taking the mathematical derivative of $s_{1}$. In contrast to the literature with traditional SMC method, for example [13], the proposed CTSMC method based scheme simplifies the design process of the controller.

Remark 4 In the proposed hierarchical control scheme for homing phase, the translational dynamics (the outer-loop) are controlled under the asymptotic stability control scheme while the finite-time convergence control scheme is adopted for the rotational dynamics (the inner-loop). The reason mainly lies in two aspects. On one hand, in contrast to asymptotic stable system, finite-time convergent system has superiorities as fast convergence rate and good anti-disturbance performance [38,39], which are highly desirable for helicopter systems landing in harsh sea conditions. On other hand, due to the underactuated property of the helicopter, the desired signals $\phi_{d}, \theta_{d}$ are generated from the position tracking controller in the outer-loop and are then fed into the inner-loop as given reference signals, so $\phi_{d}$ and $\theta_{d}$ must be at least 2nd order differentiable. However, finite-time control is essentially discontinuous control, which mean$\mathrm{s}$ that the controller is discontinuous (such as traditional discontinuous TSMC method), or nonsmooth control, namely, the controller is continuous but non-differentiable (such as the finite-time control given in Lemma 5 and the proposed CTSMC method in this paper). With this in mind, the finite-time control is only adopted in the inner-loop while the asymptotic control is developed in the outer-loop. Since the 


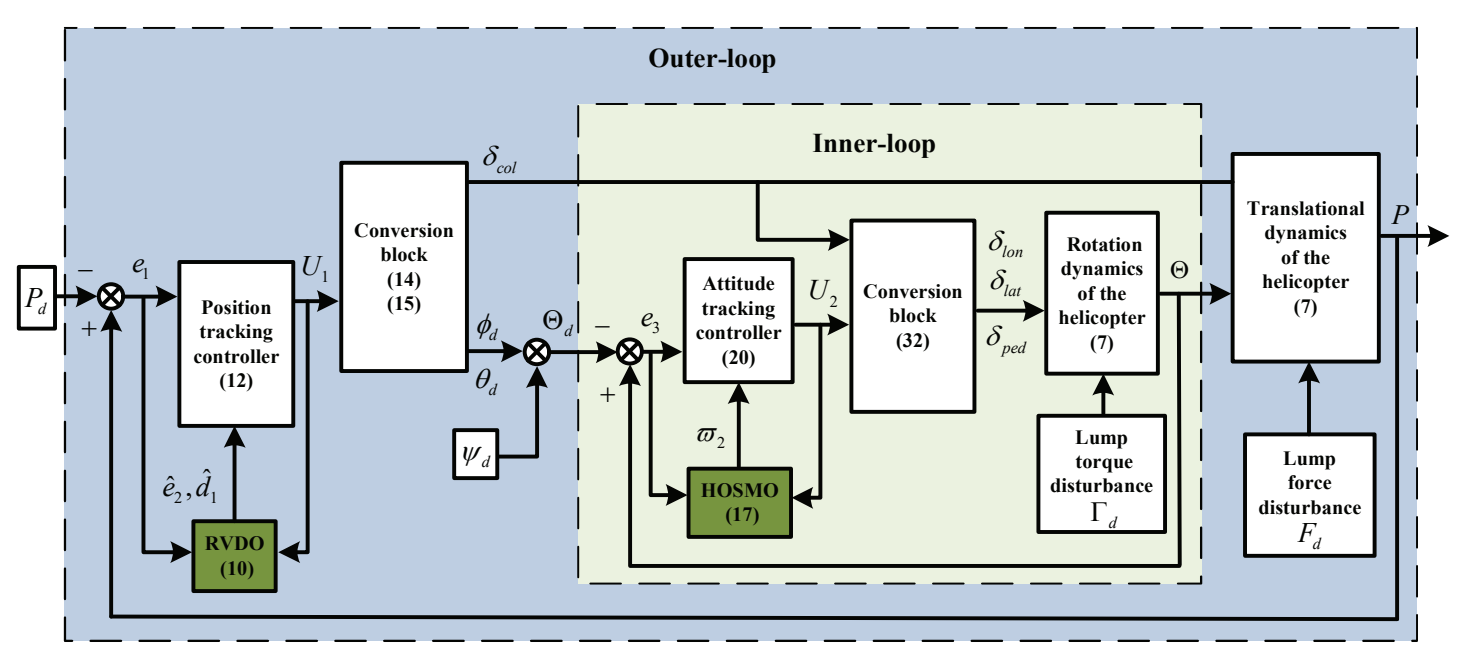

Fig. 3 The hierarchical control structure in homing phase

translational dynamics (the outer-loop) runs at an order of magnitude slower than the rotational dynamics (the inner-loop) [21], such design also meets the dynamic response speed requirements of the hierarchical control system.

\subsection{Landing phase}

In landing phase, the goal is to design the landing controller $U$ such that the helicopter lands vertically on the shipboard in synchronization with its attitudes. During the landing period, only attitude angles $\Theta=$ $[\phi, \theta, \psi]^{T}$ and vertical position $z$ are considered while the dynamics of lateral and longitudinal positions $x, y$ are ignored.

Let $\Upsilon=\left[\Theta^{T}, z\right]^{T} \in \mathbb{R}^{4}, \Xi=\left[\Omega^{T}, w\right]^{T} \in \mathbb{R}^{4}$. Based on the helicopter model (7), the landing dynamic system is expressed as

$$
\left\{\begin{array}{l}
\dot{\Upsilon}=G \Xi \\
\dot{\Xi}=F_{l}+U_{l}+d_{l}
\end{array}\right.
$$

where $G=\left[\begin{array}{cc}H & 0 \\ 0 & 1\end{array}\right], F_{l}=\left[\begin{array}{c}-J^{-1} \Omega \times J \Omega+A \Omega \\ 0\end{array}\right], U_{l}=\left[\begin{array}{c}B U \\ g+C_{\phi} C_{\theta}\left(-g+Z_{w} w+Z_{c o l} \delta_{c o l}\right)\end{array}\right], d_{l}=\left[\begin{array}{c}d_{\Omega} \\ d_{V, 3}\end{array}\right]$, $d_{V, 3}$ denotes the third row of $d_{V}$.

Take $\Upsilon_{s}=\left[\phi_{s}, \theta_{s}, \psi_{s}, z_{s}\right]^{T}$ as the attitude angles and vertical position of the shipboard. Let $e_{5}=$ $\left[e_{5, \phi}, e_{5, \theta}, e_{5, \psi}, e_{5, z}\right]^{T}=\Upsilon-\Upsilon_{s}$ represent the landing tracking error, and $e_{6}=G \Xi-\dot{\Upsilon}_{s}$. The landing tracking error system is then given by

$$
\left\{\begin{array}{l}
\dot{e}_{5}=e_{6} \\
\dot{e}_{6}=f_{2}+U_{3}+d_{3}
\end{array}\right.
$$

where $f_{2}=\dot{G} \Xi+G F_{l}, U_{3}=G U_{l}$, and $d_{3}=G d_{l}-\ddot{Y}_{s}$.

Similar to subsection 4.1.2 above, a HOSMO is designed to estimate $e_{6}$ and $d_{3}$ in (34). By combining HOSMO and the CTSMC method, a composite landing scheme is proposed. The detailed design process includes HOSMO design and composite landing controller design.

Assumption 4 For the landing tracking error system (34), the lump disturbances $d_{3, i}, i \in\{\phi, \theta, \psi, z\}$ satisfy $\left|\dot{d}_{3, i}\right| \leq M_{i}$, where $M_{i}$ are known constants. 


\section{HOSMO design}

Based on Assumption 4, the following HOSMO is designed to estimate $e_{6}$ and $d_{3}$ in (34),

$$
\left\{\begin{array}{l}
\dot{\chi}_{1}=\mu_{1}, \mu_{1}=-l_{1} M^{\frac{1}{3}} \operatorname{sig}^{\frac{2}{3}}\left(\chi_{1}-e_{5}\right)+\chi_{2}, \\
\dot{\chi}_{2}=f_{2}+U_{3}+\mu_{2}, \mu_{2}=-l_{2} M^{\frac{1}{2}} \operatorname{sig}^{\frac{1}{2}}\left(\chi_{2}-\mu_{1}\right)+\chi_{3}, \\
\dot{\chi}_{3}=-l_{3} M \operatorname{sgn}\left(\chi_{3}-\mu_{2}\right),
\end{array}\right.
$$

where $\chi_{1}, \chi_{2}$, and $\chi_{3}$ are the estimates of $e_{5}, e_{6}$, and $d_{3}$, respectively. $M=\operatorname{diag}\left\{M_{\phi}, M_{\theta}, M_{\psi}, M_{z}\right\}$, $l_{1}, l_{2}, l_{3} \in \mathbb{R}^{4 \times 4}$ are observer gains to be determined, which are all positive definite diagonal matrices.

Take the estimation errors as $\varsigma_{1}=\chi_{1}-e_{5}, \varsigma_{2}=\chi_{2}-e_{6}$, and $\varsigma_{3}=\chi_{3}-d_{3}$, respectively. Then, the estimation error dynamics are

$$
\left\{\begin{array}{l}
\dot{\zeta}_{1}=-l_{1} M^{\frac{1}{3}} \operatorname{sig}^{\frac{2}{3}}\left(\varsigma_{1}\right)+\varsigma_{2}, \\
\dot{\zeta}_{2}=-l_{2} M^{\frac{1}{2}} \operatorname{sig}^{\frac{1}{2}}\left(\varsigma_{2}-\dot{\zeta}_{1}\right)+\varsigma_{3} \\
\dot{\zeta}_{3}=-l_{3} M \operatorname{sgn}\left(\varsigma_{3}-\dot{\zeta}_{2}\right)-\dot{d}_{3}
\end{array}\right.
$$

Similarly, the estimation error system (36) is proved to be finite-time stable based on [28,29] provided that the observer scaler gains $M_{i} \geq\left|\dot{d}_{3, i}\right|, i \in\{\phi, \theta, \psi, z\}$. This means that all signals in (36) are bounded and there exists a finite time constant $T_{l}>0$ such that $\varsigma_{1} \equiv 0, \varsigma_{2} \equiv 0$, and $\varsigma_{3} \equiv 0$, for $t \geq T_{l}$.

II. Composite landing controller design

A novel dynamic output terminal sliding manifold $s_{2}$ is designed as

$$
s_{2}=K_{5} \operatorname{sig}^{\alpha_{3}}\left(e_{5}\right)+K_{6} \operatorname{sig}^{\alpha_{4}}\left(\hat{e}_{6}\right)+f_{2}+U_{3}+\hat{d}_{3},
$$

where $K_{5}$ and $K_{6}$ are positive definite diagonal matrices. $\alpha_{3}=\left[\alpha_{3, \phi}, \alpha_{3, \theta}, \alpha_{3, \psi}, \alpha_{3, z}\right]^{T}, \alpha_{4}=\left[\alpha_{4, \phi}, \alpha_{4, \theta}, \alpha_{4, \psi}, \alpha_{4, z}\right]^{T}$, and $0<\alpha_{3, i}<1, \alpha_{4, i}=\frac{2 \alpha_{3, i}}{1+\alpha_{3, i}}, i \in\{\phi, \theta, \psi, z\} . \hat{e}_{6}$ and $\hat{d}_{3}$ represent, respectively, the estimates of $e_{6}$ and $d_{3}$, namely, $\hat{e}_{6}=\chi_{2}, \hat{d}_{3}=\chi_{3}$.

Design $U_{3}=\left[U_{3, \phi}, U_{3, \theta}, U_{3, \psi}, U_{3, z}\right]^{T}$ as

$$
\left\{\begin{array}{l}
U_{3}=-\left(U_{3}^{e q}-U_{3}^{n}\right) \\
U_{3}^{e q}=K_{5} \operatorname{sig}^{\alpha_{3}}\left(e_{5}\right)+K_{6} \operatorname{sig}^{\alpha_{4}}\left(\hat{e}_{6}\right)+f_{2}+\hat{d}_{3} \\
\dot{U}_{3}^{n}=-\eta_{2} \operatorname{sgn}\left(s_{2}\right)
\end{array}\right.
$$

where $\eta_{2} \in \mathbb{R}^{4 \times 4}$ is a positive definite diagonal matrix.

With the same argument, the inverse of $G$ is $G^{-1}=\left[\begin{array}{cc}H^{-1} & 0 \\ 0 & 1\end{array}\right]$. By some algebraic transformations, the landing controller $U=\left[\delta_{c o l}, \delta_{l o n}, \delta_{l a t}, \delta_{p e d}\right]^{T}$ is computed as

$$
\left\{\begin{array}{l}
\delta_{c o l}=\frac{U_{3, z}-g}{C_{\phi} C_{\theta} Z_{c o l}}+\frac{g-Z_{w} w}{Z_{c o l}}, \\
\delta_{l o n}=\frac{\left(C_{\phi} U_{3, \theta}+S_{\phi} C_{\theta} U_{3, \psi}\right) L_{l a t}-\left(U_{3, \phi}-S_{\theta} U_{3, \psi}\right) M_{l a t}}{L_{l a t} M_{l o n}-L_{l o n} M_{l a t}}, \\
\delta_{l a t}=\frac{\left(U_{3, \phi}-S_{\theta} U_{3, \psi}\right) M_{l o n}-\left(C_{\phi} U_{3, \theta}+S_{\phi} C_{\theta} U_{3, \psi}\right) L_{l o n}}{L_{l a t} M_{l o n}-L_{l o n} M_{l a t}}, \\
\delta_{\text {ped }}=\frac{-S_{\phi} U_{3, \theta}+C_{\phi} C_{\theta} U_{3, \psi}}{N_{p e d}}-\frac{N_{c o l}\left(U_{3, z}-g\right)}{N_{p e d} C_{\phi} C_{\theta} Z_{c o l}}-\frac{N_{c o l}\left(g-Z_{w} w\right)}{N_{p e d} Z_{c o l}}
\end{array} .\right.
$$


Theorem 3 For the landing tracking error system (34) under the landing controller (39) with the HOSMO (35), if the observer scaler gains $M_{i}, i \in\{\phi, \theta, \psi, z\}$ in (35) are chosen such that $M_{i} \geq\left|\dot{d}_{3, i}\right|$, then the closed-loop landing tracking error system (34) and (38) is finite-time convergent, that is, the landing tracking error $e_{5}$ converges to zero in finite time.

Proof The proof of Theorem 3 is similar to that of Theorem 2, so it is omitted here.

Remark 5 In homing phase, all states of the helicopter are considered such that the helicopter (7) is an underactuated 6-DOF system with four control inputs. Because of the underactuated property, a hierarchical double-loop control scheme is developed. For the reasons mentioned in Remark 4, the outer-loop adopts asymptotic stability control scheme by combining a RVDO with relative position feedback control, while the inner-loop adopts finite-time convergence control scheme by combining a HOSMO with CTSMC method. In landing phase, since the landing process is fast, only the degrees of freedom of attitude angles and altitude are considered. Hence, the landing dynamic system (33) is a fully actuated 4-DOF system with four control inputs. To this end, the landing control scheme does not need to adopt hierarchical double-loop control, but can be the same as the inner-loop scheme in homing phase, that is, a composite landing scheme with the integration of a HOSMO and CTSMC method.

\section{Simulations Studies}

To show the effectiveness and the superiorities of the proposed composite landing control scheme, numerical simulations are performed in this section.

The model parameters of the helicopter are given as follows [40] : $g=9.81 \mathrm{~m} \cdot \mathrm{s}^{-2}, m=8.2 \mathrm{~kg}, Z_{w}=$ $-0.7615 \mathrm{~s}^{-1}, Z_{c o l}=-131.4125 \mathrm{~m} /\left(\mathrm{rad} \cdot \mathrm{s}^{2}\right), J=\operatorname{diag}\{0.18,0.34,0.28\} \mathrm{kg} \cdot \mathrm{m}^{2}, A=\operatorname{diag}\{-48.1757,-25.5048,-0.9808\} \mathrm{s}$ and

$$
B=\left[\begin{array}{cccc}
0 & 0 & 1689.5 & 0 \\
0 & 894.5 & 0 & 0 \\
-0.3705 & 0 & 0 & 135.8
\end{array}\right] \mathrm{s}^{-2} .
$$

The lump disturbances $F_{d}$ and $\Gamma_{d}$ are set as

$$
\begin{gathered}
F_{d}=\left[\begin{array}{l}
1+\sin (0.4 t) \\
1+\sin (0.2 t) \\
1+\sin (0.2 t)
\end{array}\right] \mathrm{kg} \cdot \mathrm{m} \cdot \mathrm{s}^{-2}, \\
\Gamma_{d}=\left[\begin{array}{l}
0.8+\sin (0.01 t)+\sin (0.2 t) \\
0.8+\sin (0.01 t)+\sin \left(\frac{\pi}{15} t\right) \\
0.8+\sin (0.01 t)+\sin \left(\frac{\pi}{15} t\right)
\end{array}\right] \mathrm{kg} \cdot \mathrm{m}^{2} \cdot \mathrm{rad} \cdot \mathrm{s}^{-2} .
\end{gathered}
$$

\subsection{Simulations in homing phase}

In this subsection, numerical simulation is carried out for homing phase. The initial conditions of the helicopter are set as $P(0)=[6,6,6]^{T} \mathrm{~m}$ and $\psi(0)=\frac{\pi}{12} \mathrm{rad}$. The initial values of other state elements in (7) are all set to be zero. The desired hovering altitude and yaw angle are $z_{d}=1 \mathrm{~m}$ and $\psi_{d}=0 \mathrm{rad}$. The motions of the shipboard are considered as $x_{s}=1.3 \sin (0.4 t) \mathrm{m}$ and $y_{s}=2 \sin (0.2 t) \mathrm{m}$.

According to the mathematical form of $F_{d}$ and $x_{s}$, it is known that the disturbance $d_{1, x}$ in (8) includes one harmonic component with frequency 0.4 and one constant component, namely, $m_{x}=1, \omega_{1, x}=$ $0.4, n_{x}=1$. Similarly, it can be obtained that $m_{y}=m_{z}=1, \omega_{1, y}=\omega_{1, z}=0.2, n_{y}=n_{z}=1$. Hence, $\Phi_{x}, \Phi_{y}, \Phi_{z}$ in $(11) \in \mathbb{R}^{4 \times 4}$ and the observer gains of the RVDO (10) are chosen as $a_{0, x}=12, a_{1, x}=-452.41, a_{2, x}=$ 


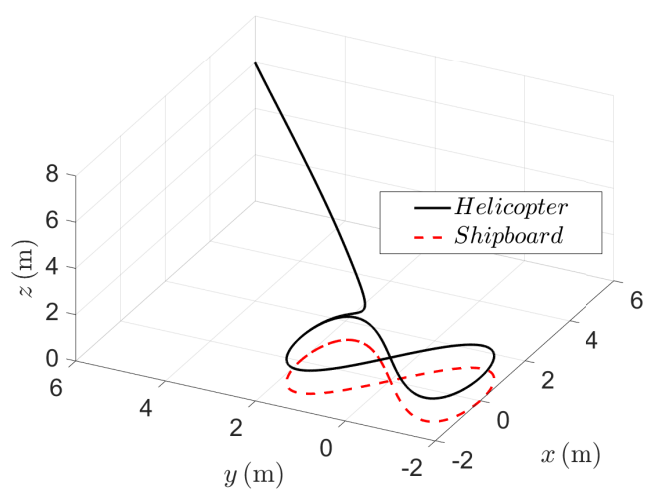

Fig. 4 Three-dimensional trajectory of the helicopter and shipboard in homing phase

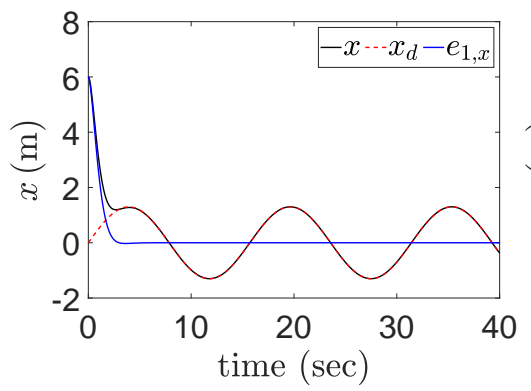

(a)

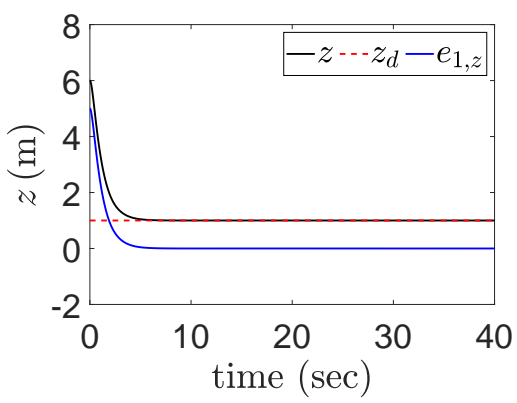

(c)

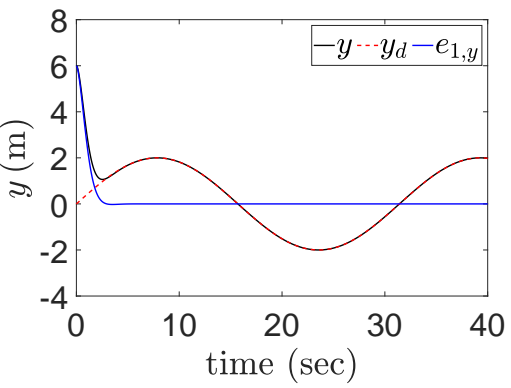

(b)

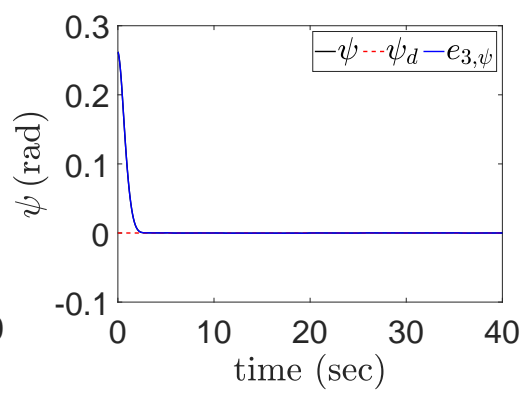

(d)

Fig. 5 Response curves of the positions and yaw angle in homing phase (a) Lateral position $x$. (b) Longitudinal position $y$. (c) Vertical position $z$. (d) Yaw angle $\psi$.

$106.08, b_{1, x}=506.25, a_{0, y}=a_{0, z}=12, a_{1, y}=a_{1, z}=-1971.04, a_{2, y}=a_{2, z}=107.52, b_{1, y}=b_{1, z}=2025$, which are obtained by assigning the fourfold poles of estimation error characteristic polynomial as -3 . The controller gains in (12) are $K_{1}=\operatorname{diag}\{3,3,4\}$ and $K_{2}=\operatorname{diag}\{3,3,3\}$. The observer gains of the HOSMO (17) are $L=\operatorname{diag}\{4,4,4\}, \lambda_{1}=\operatorname{diag}\{1.5,1.5,1.5\}$, and $\lambda_{2}=\operatorname{diag}\{1,1,1\}$. The controller gains in (20) are $K_{3}=\operatorname{diag}\{4,4,4\}, K_{4}=\operatorname{diag}\{4,4,4\}, \alpha_{1}=\left[\frac{8}{11}, \frac{8}{11}, \frac{8}{11}\right]^{T}$, and $\eta_{1}=\operatorname{diag}\{3,3,3\}$.

Figs. 4-8 demonstrate the simulation results. Fig. 4 depicts the three-dimensional trajectories of the helicopter and shipboard in homing phase. Fig. 5 gives the positions and yaw angle response curves of the helicopter under the proposed homing control scheme. It can be observed from Figs. 4 and 5 that, under the homing controller, the positions and yaw angle of the helicopter converge to the desired values asymptotically even in the presence of lump disturbances. The time history of the homing controller with 


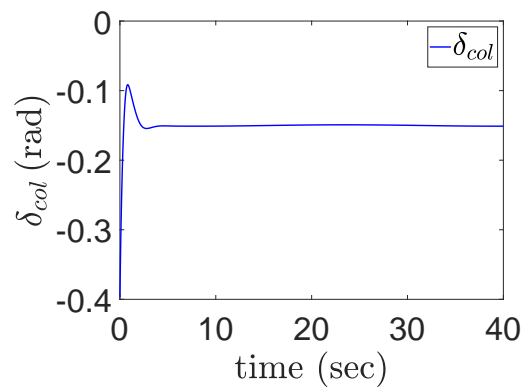

(a)

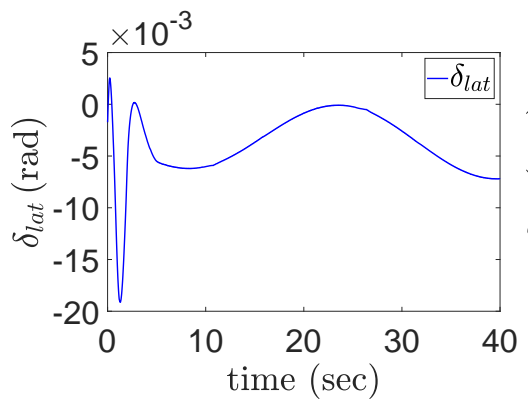

(c)

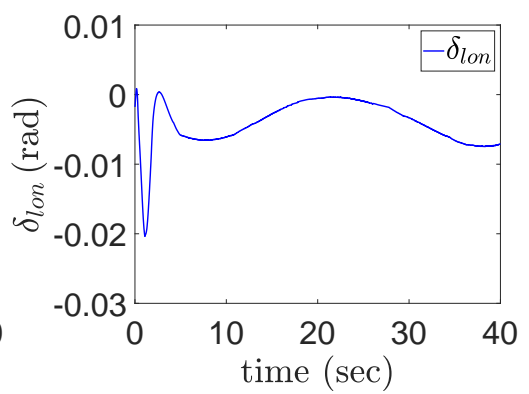

(b)

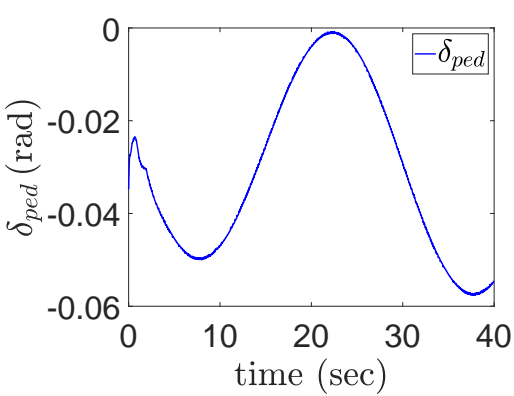

(d)

Fig. 6 Time history of the controller in homing phase (a) $\delta_{c o l}$. (b) $\delta_{l o n}$. (c) $\delta_{\text {lat }}$. (d) $\delta_{\text {ped }}$.

four control inputs is shown in Fig. 6, which shows that the control inputs are continuous. The estimation errors on $e_{2}$ and $d_{1}$ of the RVDO (10) are provided in Fig. 7. From Fig. 7, it is shown that both the estimation errors of the system state and the disturbance converge to zero asymptotically, which indicates that the velocity and acceleration of the shipboard as well as the disturbance can be accurately estimated by the observer. Fig. 8 depicts the estimation errors of $d_{2}$ from the HOSMO (17), which can be seen to converge to zero in finite time.

\subsection{Simulations in landing phase}

In this subsection, three simulations are conducted to illustrate the effectiveness and the superiorities of the proposed landing scheme. The first simulation is conducted under the proposed CTSMC and HOSMO based scheme (38) and (35). By taking $\hat{d}_{3}$ in (37) and (38) as zero, the second simulation is performed under the CTSMC based scheme (38) and (35). The third simulation is conducted based on the traditional discontinuous SMC method in [14], where the controller is presented as follows

$$
\left\{\begin{array}{l}
s_{3}=\hat{e}_{6}+a_{1} e_{5}, \\
U_{3}=-f_{2}-\mu_{2}-a_{1} \hat{e}_{6}-a_{2} \operatorname{sgn}\left(s_{3}\right),
\end{array}\right.
$$

where $a_{1}$ and $a_{2}$ are positive definite diagonal matrices. $e_{5}$ and $f_{2}$ are from (34), $\hat{e}_{6}$ and $\mu_{2}$ are from the HOMSO (35). Under the compared controller (40) and (35), the landing tracking error $e_{5}$ converges to zero asymptotically. For convenience, the three schemes are abbreviated as CTSMC+HOSMO, CTSMC, and SMC+HOSMO, respectively.

In these three simulations, the initial conditions of the helicopter are all set as $\Psi(0)=\left[\frac{\pi}{12}, \frac{\pi}{12}, \frac{\pi}{12}\right]^{T} \mathrm{rad}$ and $z(0)=1 \mathrm{~m}$. The initial values of other state elements in (33) are all set to be zero. The attitude angles 


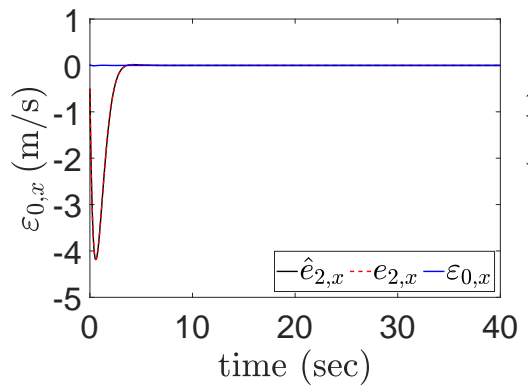

(a)

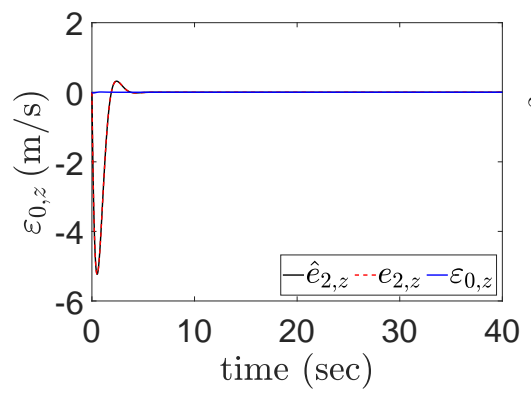

(c)

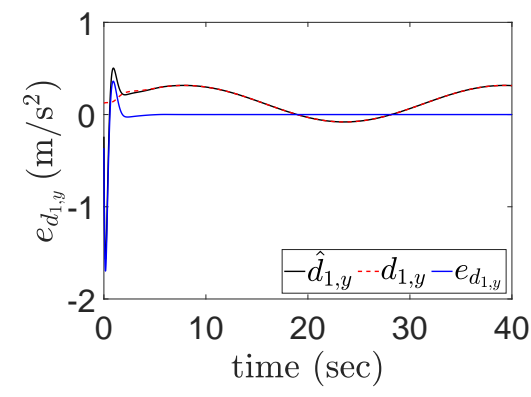

(e)

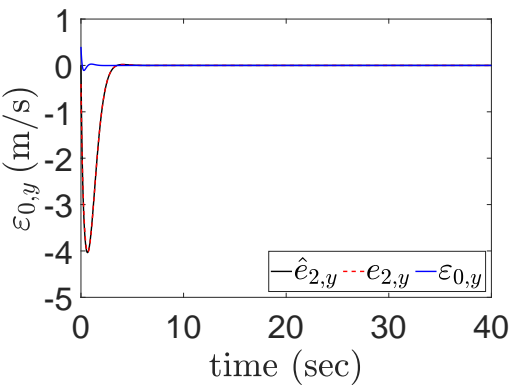

(b)

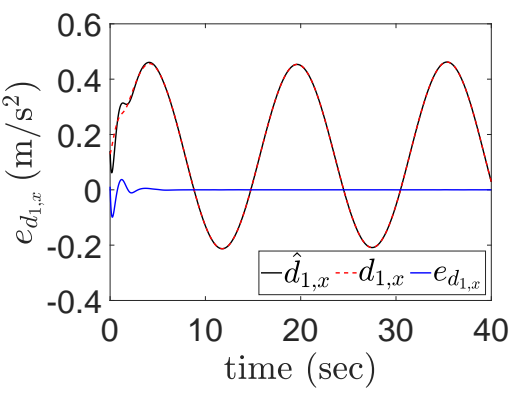

(d)

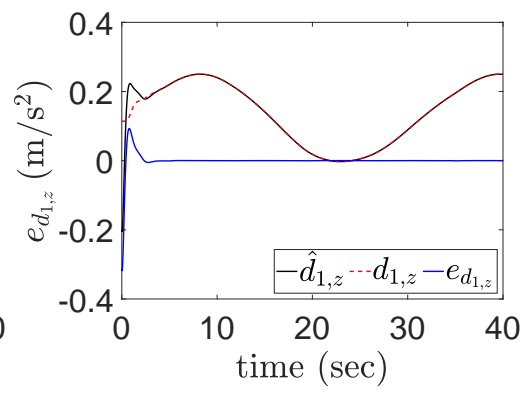

(f)

Fig. 7 Estimation error curves of the RVDO (10) (a) $\sigma_{0, x}$ (estimation error of $e_{2, x}$ ). (b) $\sigma_{0, y}$ (estimation error of $e_{2, y}$ ). (c) $\sigma_{0, z}$ (estimation error of $e_{2, z}$ ). (d) $e_{d_{1, x}}=\hat{d}_{1, x}-d_{1, x}$. (e) $e_{d_{1, y}}=\hat{d}_{1, y}-d_{1, y}$. (f) $e_{d_{1, z}}=\hat{d}_{1, z}-d_{1, z}$.

and vertical position of the shipboard are all considered as $\Psi_{s}(0)=[0.15 \sin (0.25 t), 0.15 \sin (0.25 t), 0.15 \sin (0.4 t)]^{T} \operatorname{rad}$ and $z_{s}(0)=0.5 \sin (0.15 t) \mathrm{m}$.

To conduct fair comparison, the control inputs of these three simulations should be at the same levels. Based on this principle, the observer gains of HOSMO (35) in these three simulations are al1 set as $M=\operatorname{diag}\{7,7,7,7\}, l_{1}=\operatorname{diag}\{3,3,3,3\}, l_{2}=\operatorname{diag}\{1.5,1.5,1.5,1.5\}$, and $l_{3}=\operatorname{diag}\{1,1,1,1\}$. For CTSMC+HOSMO scheme, the controller gains in (38) are chosen as $K_{5}=\operatorname{diag}\{10,10,10,10\}$, $K_{6}=\operatorname{diag}\{10,10,10,10\}, \alpha_{3}=\left[\frac{5}{7}, \frac{5}{7}, \frac{5}{7}, \frac{5}{7}\right]^{T}$, and $\eta_{2}=\operatorname{diag}\{2,2,2,2\}$. For CTSMC scheme, the controller gains in (38) are chosen as $K_{5}=\operatorname{diag}\{20,20,20,20\}, K_{6}=\operatorname{diag}\{20,20,20,20\}, \alpha_{3}=\left[\frac{5}{7}, \frac{5}{7}, \frac{5}{7}, \frac{5}{7}\right]^{T}$, and $\eta_{2}=\operatorname{diag}\{2,2,2,2\}$. For SMC+HOSMO scheme, the controller gains in (40) are chosen as $a_{1}=$ $\operatorname{diag}\{5,5,5,5\}$ and $a_{2}=\operatorname{diag}\{5,5,5,5\}$.

The simulation results are shown in Figs. 9-12. Figs. 9 and 10 demonstrate the attitude angles and vertical position response curves of the helicopter in three simulations. It is observed from the curves under CTSMC+HOSMO and CTSMC schemes that, the landing error curves under the CTSMC+HOSMO 


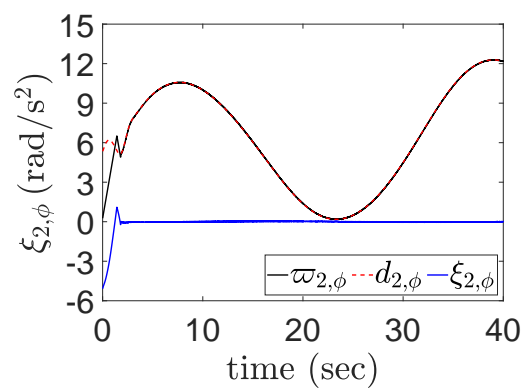

(a)

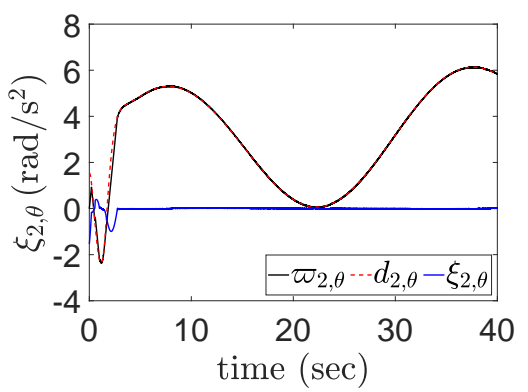

(b)

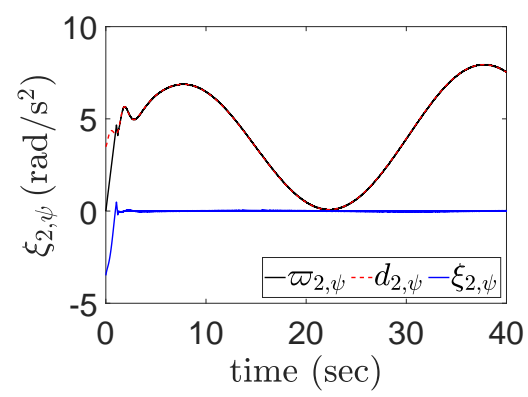

(c)

Fig. 8 Estimation error curves of the $\operatorname{HOSMO}$ (17) (a) $\xi_{2, \phi}$ (estimation error of $d_{2, \phi}$ ). (b) $\xi_{2, \theta}$ (estimation error of $d_{2, \theta}$ ). (c) $\xi_{2, \psi}$ (estimation error of $d_{2, \psi}$ ).

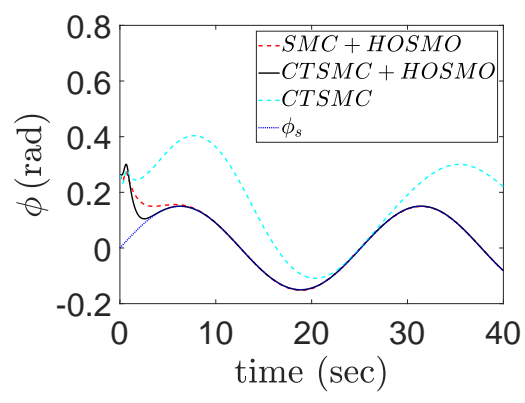

(a)

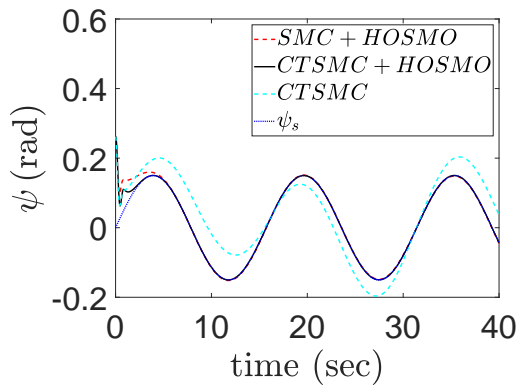

(c)

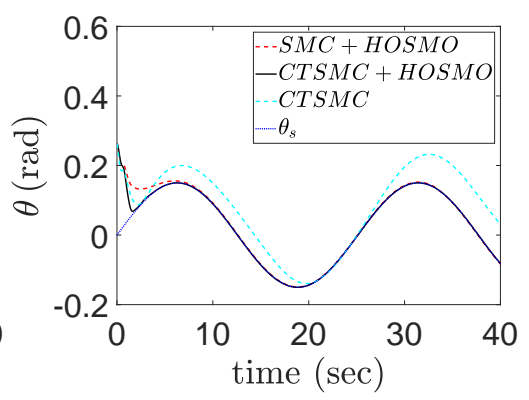

(b)

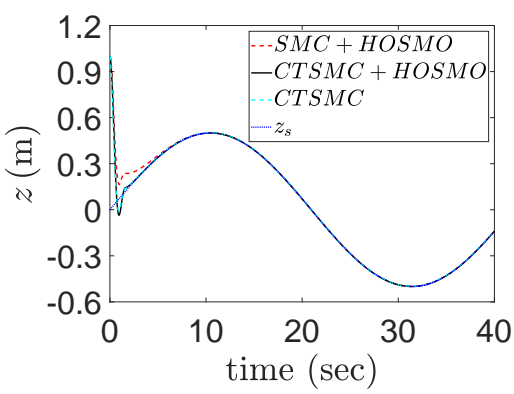

(d)

Fig. 9 Response curves of the attitude angles and vertical position under three controllers in landing phase (a) Roll angle $\phi$. (b) Pitch angle $\theta$. (c) Yaw angle $\psi$. (d) Vertical position $z$. 


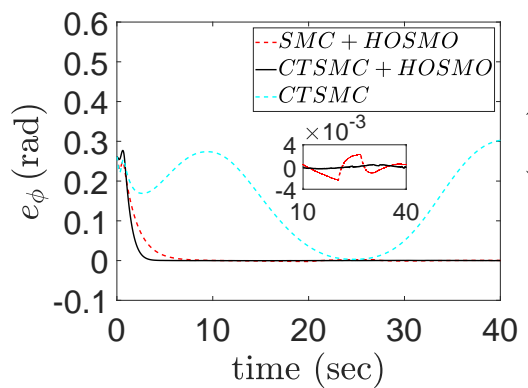

(a)

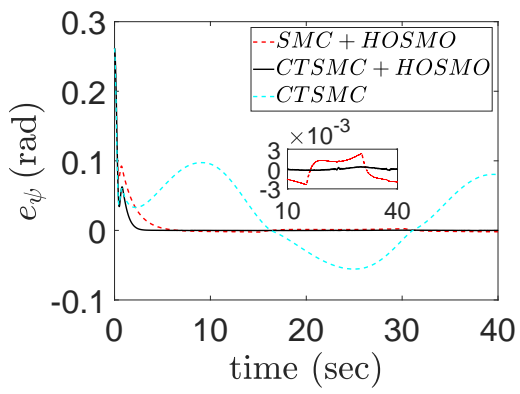

(c)

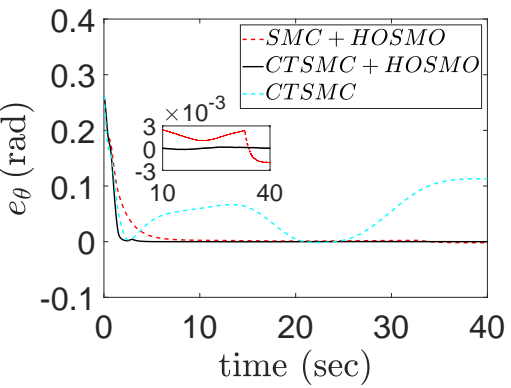

(b)

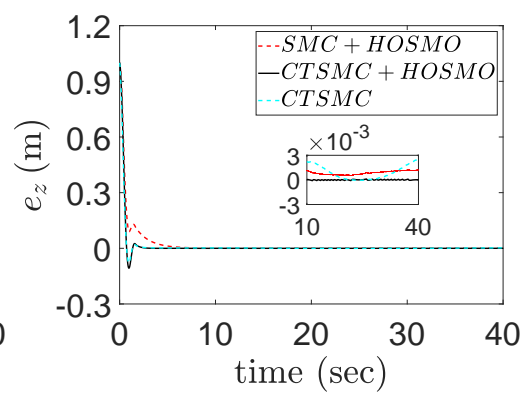

(d)

Fig. 10 Error curves of the attitude angles and vertical position under three controllers in landing phase (a) $e_{\phi}$. (b) $e_{\theta}$. (c) $e_{\psi}$. (d) $e_{z}$

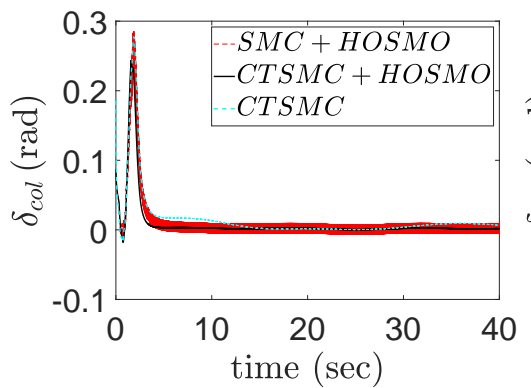

(a)

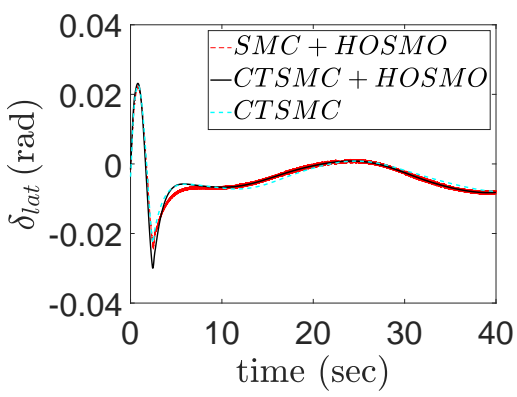

(c)

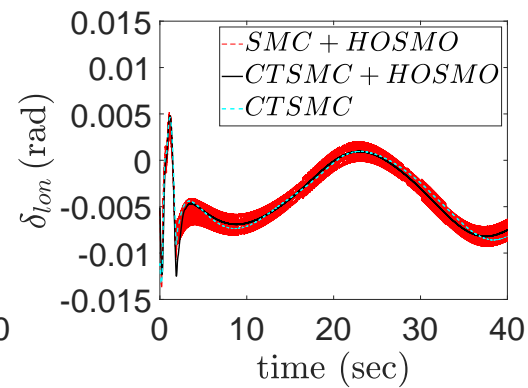

(b)

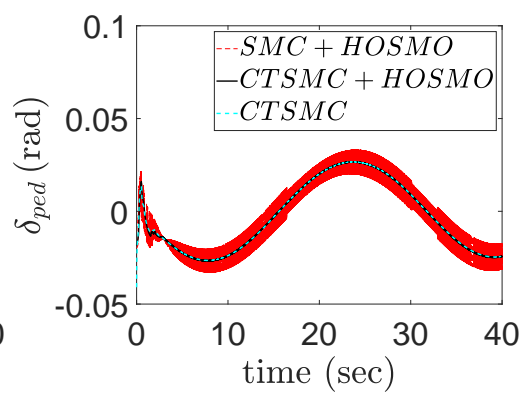

(d)

Fig. 11 Time history of the controllers in landing phase in three simulations (a) $\delta_{c o l}$. (b) $\delta_{l o n}$. (c) $\delta_{l a t}$. (d) $\delta_{p e d}$. 


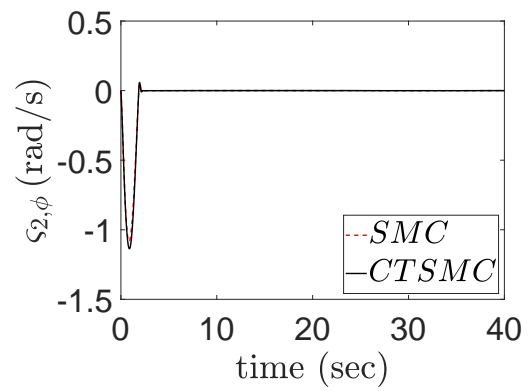

(a)

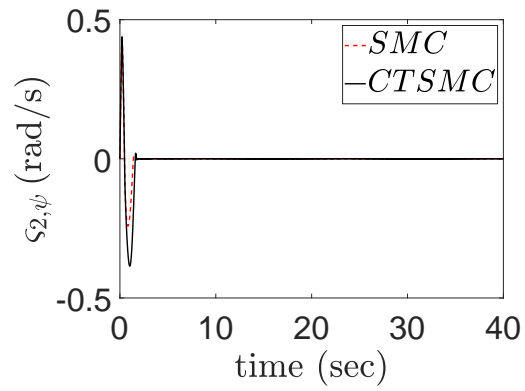

(c)

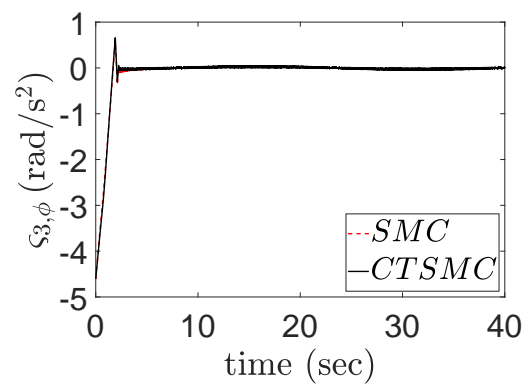

(e)

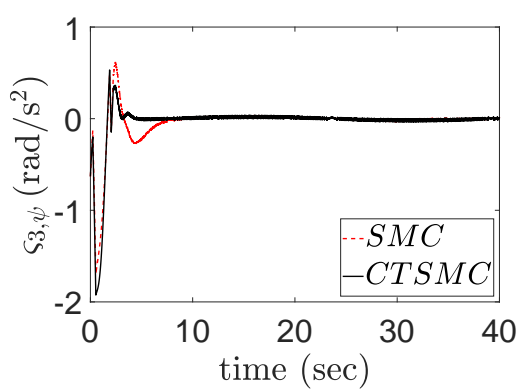

(g)

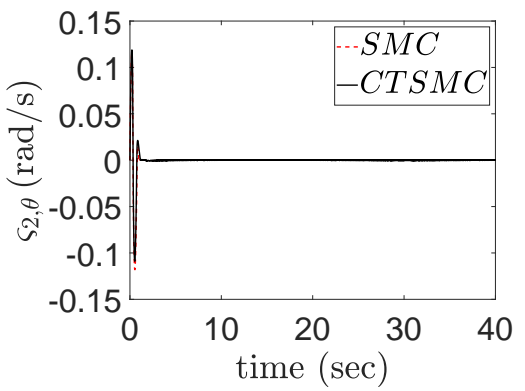

(b)

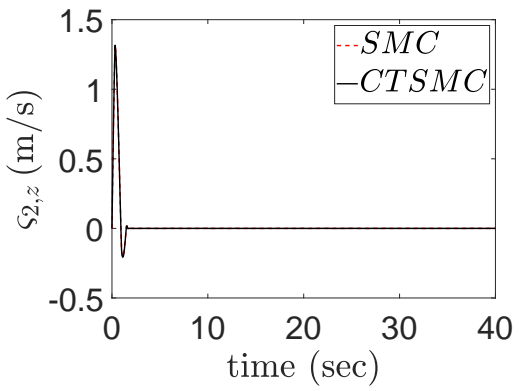

(d)

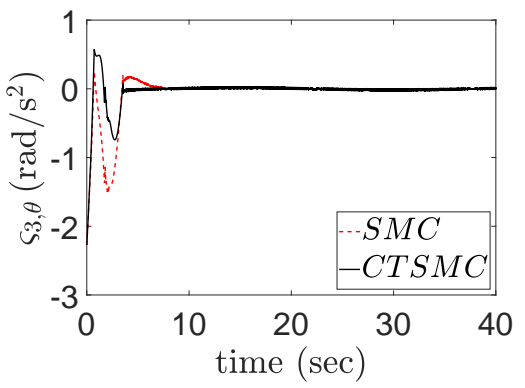

(f)

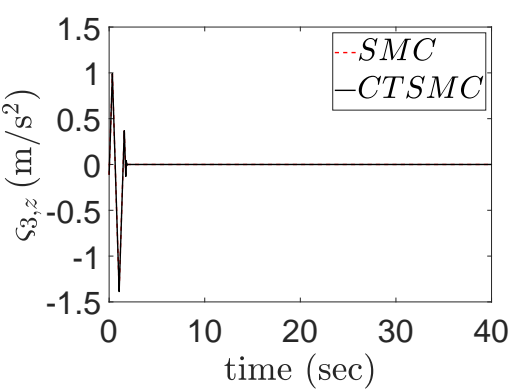

(h)

Fig. 12 Estimation error curves of the HOSMOs (35) (a) $\varsigma_{2, \phi}$ (estimation error of $e_{6, \phi}$ ). (b) $\varsigma_{2, \theta}$ (estimation error of $e_{6, \theta}$ ). (c) $\varsigma_{2, \psi}$ (estimation error of $e_{6, \psi}$ ). (d) $\varsigma_{2, z}$ (estimation error of $e_{6, z}$ ). (e) $\varsigma_{3, \phi}$ (estimation error of $d_{3, \phi}$ ). (f) $\varsigma_{3, \theta}$ (estimation error of $d_{3, \theta}$ ). (g) $\varsigma_{3, \psi}$ (estimation error of $d_{3, \psi}$ ). (h) $\varsigma_{3, z}$ (estimation error of $d_{3, z}$ ).

scheme is much smaller than those under the CTSMC scheme, which fully demonstrates the good compensation of HOMSO. From the curves under the CTSMC+HOSMO and SMC+HOSMO schemes, it 
is shown that, compared to asymptotic SMC+HOSMO scheme, finite-time CTSMC+HOSMO scheme achieves smaller landing errors of the closed-loop system, which clearly shows the superiorities of the finite-time control scheme. Fig. 11 depicts the time histories of the landing controllers under three schemes. It is observed that the control action are discontinuous under the SMC+HOSMO scheme, while they are being continuous under the proposed CTSMC+HOSMO and the compared CTSMC schemes. This fully verifies that the proposed CTSMC method effectively mitigates the chattering problem caused by switching functions in traditional SMC method. The estimation error curves on $e_{6}$ and $d_{3}$ of the HOSMO (35) are shown in Fig. 12, which indicates that the estimation errors of the system state and the disturbance converge to zero in finite time.

\section{Conclusions}

The autonomous landing control problem on moving shipboard has been investigated for disturbed helicopters in this paper. By dividing the whole landing process into homing phase and landing phase, the helicopter has been forced to land on a moving shipboard from a distant location. The velocity and acceleration information of the shipboard have been estimated along with lump disturbances through the RVDO/HOSMO such that the errors caused by the unmodeled shipboard dynamics and inaccurate state measurements are effectively avoided, which is one of the major contribution of this paper. Based on the suppression from CTSMC method and feedforward compensation of the disturbance estimates from RVDO/HOSMO, the proposed composite landing scheme has provided double guarantees for the anti-disturbance performance and robustness of the closed-loop system. In addition, the proposed control method mitigates the chattering of the control action as well as simplifying the design process of the controller.

The limitation of this work exists on treating various factors affecting the helicopter system as lumped disturbances, which may lead to insufficient targeted compensation for these factors. Future works will be concentrated on detailed modeling and analysis of these factors, as well as the design of targeted disturbance observation and compensation schemes.

Acknowledgements The work was supported in part by the National Natural Science Foundation of China under Grant 61973080 and Grant 61973081, in part by the Shenzhen Science and Technology Innovation Committee (STIC) under Grant JCYJ20190813152603594.

\section{Conflict of interest statement}

There is no conflict of interest.

\section{References}

1. Shin, H., You, D., Shim, D.H.: Autonomous shipboard landing algorithm for unmanned helicopters in crosswind. J. Intell. Robot. Syst. 74(1-2), 347-361 (2014)

2. Jiang, J., Qi, Y., Ibrahim, M., et al.: Quadrotors' low-cost vision-based autonomous landing architecture on a moving platform. In: 2018 15th International Conference on Control, Automation, Robotics and Vision, Singapore, pp. 448-453 (2018)

3. Chen, X., Phang, S.K., Shan, M., et al.: System integration of a vision-guided UAV for autonomous landing on moving platform. In: 2016 12th IEEE International Conference on Control and Automation, Kathmandu, Nepal, pp. 761-766 (2016)

4. Deng, Y., Duan, H.: Control parameter design for automatic carrier landing system via pigeon-inspired optimization. Nonlinear Dyn. 85(1), 97-106 (2016)

5. Li, J., Duan, H.: Simplified brain storm optimization approach to control parameter optimization in F/A-18 automatic carrier landing system. Aerosp. Sci. Technol. 42, 187-195 (2015)

6. Ali, U., Shah, M.Z., Samar, R., et al.: Robust level flight control design for scaled Yak-54 unmanned aerial vehicle using single sliding surface. In: 2012 24th Chinese Control and Decision Conference, Taiyuan, China, pp. 1209-1214 (2012) 
7. Saripalli, S., Sukhatme, G. (2006). Landing on a moving target using an autonomous helicopter. In: Field and Service Robotics, pp. 277C286. Springer.

8. Hoffmann, G.M., Huang, H., Waslander, S.L, et al.: Precision flight control for a multi-vehicle quadrotor helicopter testbed. Control Eng. Pract., 19(9), 1023-1036 (2011)

9. Venugopalan, T.K., Taher, T., Barbastathis, G.: Autonomous landing of an unmanned aerial vehicle on an autonomous marine vehicle. In: 2012 Oceans, Yeosu, Korea, pp. 1-9 (2012)

10. Subrahmanyam, M.B.: H-infinity design of F/A-18A automatic carrier landing system. J. Guid. Control Dyn. 17(1), 187-191 (1994)

11. Lungu, R., Lungu, M.: Design of automatic landing systems using the H-inf control and the dynamic inversion. J. Dyn. Syst. Meas. Control 138(2), (2016)

12. Ghommam, J., Saad, M.: Autonomous landing of a quadrotor on a moving platform. IEEE Trans. Aerosp. Electron. Syst. 53(3), 1504-1519 (2017)

13. Guan, Z., Ma, Y., Zheng, Z., et al: Prescribed performance control for automatic carrier landing with disturbance. Nonlinear Dyn. 94(2), 1335-1349 (2018)

14. Zheng, Z., Jin, Z., Sun, L., et al.: Adaptive sliding mode relative motion control for autonomous carrier landing of fixed-wing unmanned aerial vehicles. IEEE Access 5, 5556-5565 (2017)

15. Huang, Y., Zhu, M., Zheng, Z., et al.: Fixed-time autonomous shipboard landing control of a helicopter with external disturbances. Aerosp. Sci. Technol. 84, 18-30 (2019)

16. Rao, D.V, Go, T.H.: Automatic landing system design using sliding mode control. Aerosp. Sci. Technol. 32(1), 180-187 (2014)

17. Malaek, S.M.B., Sadati, N., Izadi, H., et al.: Intelligent autolanding controller design using neural networks and fuzzy logic. In: 5th Asian Control Conference, pp. 365-373 (2004)

18. Zhao, Z., Yang, J., Li, S., et al.: Continuous output feedback TSM control for uncertain systems with a DC-AC inverter example. IEEE Trans. Circuits Syst. II-Express Briefs. 65(1), 71-75 (2018)

19. Wang, L., Mishra, J., Zhu Y., et al.: An improved sliding-mode current control of induction machine in presence of voltage constraints. IEEE Trans. Ind. Inform. 16(2): 1182-1191 (2020)

20. Bagen, W., Hu, J., Xu, Y.: A vision-based unmanned helicopter ship board landing system. In: 2nd International Congress on Image and Signal Processing, pp. 1-5 (2009)

21. Oh, S.R., Pathak, K., Agrawal, S.K., et al.: Approaches for a tether-guided landing of an autonomous helicopter. IEEE Trans. Robot. 22(3), 536-544 (2016)

22. Daly, J.M, Ma, Y, Waslander, S.L.: Coordinated landing of a quadrotor on a skid-steered ground vehicle in the presence of time delays. Auton. Robot. 38(2), 179-191 (2015)

23. Wenzel, K.E, Masselli, A., Zell, A.: Automatic take off, tracking and landing of a miniature UAV on a moving carrier vehicle. J. Intell. Robot. Syst. 61(1-4), 221-238 (2011)

24. Madonski R., Ramirez-Neria M., Gao Z., et al.: Attenuation of periodic disturbances via customized ADRC solution: a case of highly oscillatory 3DOF torsional plant. In: 2019 8th Data Driven Control and Learning System Conference, Dali, China, pp. 1111-1116 (2019)

25. Wang, X., Yu, X., Li, S., et al.: Composite block backstepping trajectory tracking control for disturbed unmanned helicopters. Aerosp. Sci. Technol. 85, 386-398 (2019)

26. Yue, Y., Wang, H., Li, N., et al.: Automatic carrier landing system based on active disturbance rejection control with a novel parameters optimizer. Aerosp. Sci. Technol. 69, 149-160 (2017).

27. Chen W.H.: Disturbance observer based control for nonlinear systems. IEEE-ASME Trans. Mechatron. 9(4), 706-710 (2004)

28. Levant, A.: Higher-order sliding modes, differentiation and output-feedback control, Int. J. Control, 76(9-10), 924-941 (2003)

29. Shtessel, Y.B., Shkolnikov, I.A., Levant, A.: Smooth second-order sliding modes: Missile guidance application. Automatica 43(8), 1470-1476 (2007)

30. Madonski R., Ramirez-Neria M., Stanković M., et al.: On vibration suppression and trajectory tracking in largely uncertain torsional system: An error-based ADRC approach. Mech. Syst. Signal Pr. 134(1) (2019)

31. Madonski R., Stanković M., Shao S., et al.: Active disturbance rejection control of torsional plant with unknown frequency harmonic disturbance. Control Eng. Pract. 100 (2020)

32. Khalil, H.K. Nonlinear Systems (3rd ed.). Prentice Hall. (2002)

33. Hardy, G.H, Littlewood, J.E., Pólya, G., et al.: Inequalities. Cambridge University Press. (1952).

34. Bhat, S.P, Bernstein D.S.: Finite-time stability of continuous autonomous systems. SIAM J. Control Optim. 38(3), 751-766 (2000)

35. Bhat, S., Bernstein, D.: Finite-time stability of homogeneous systems. Am. Control Conf. 4(4), 2513-2514 (1997)

36. Liu, C., Chen, W.H., Andrews, J.: Tracking control of small-scale helicopters using explicit nonlinear MPC augmented with disturbance observers, Control Eng. Pract. 20(3), 258-268 (2012)

37. Bogdanov, A., Wan, E.A.: State-dependent riccati equation control for small autonomous helicopters, J. Guid. Control Dyn. 30(1), 47-60 (2007)

38. Yu, S., Yu, X., Shirinzadeh, B., et al.: Continuous finite-time control for robotic manipulators with terminal sliding mode. Automatica, 41(11), 1957-1964 (2005)

39. Bhat, S.P, Bernstein, D.S.: Continuous finite-time stabilization of the translational and rotational double integrators. IEEE Trans. Autom. Control 43(5), 678-682 (1998)

40. Fang, X., Wu, A., Shang, Y., et al.: A novel sliding mode controller for small-scale unmanned helicopters with mismatched disturbance. Nonlinear Dyn. 83(1-2), 1053-1068 (2016) 